\title{
Sodium-ketyl Radical Anions by Reverse Pinacol Reaction and their Coupling with Iodoarenes
}

\author{
Xinjun Tang, ${ }^{\dagger}$ Armido Studer ${ }^{* \dagger}$ \\ † Organisch-Chemisches Institut, Westfälische Wilhelms-Universität, Corrensstraße 40, 48149 \\ Münster, Germany \\ *E-mail: studer@uni-muenster.de
}

\section{Supporting information}

\section{Table of Contents}

1. Instrumentation and chemicals............................................

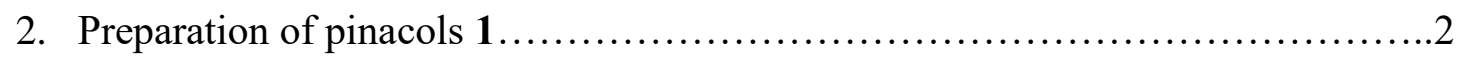

3. Reaction of iodoarenes with pinacols......................................2

4. References................................................................ 10

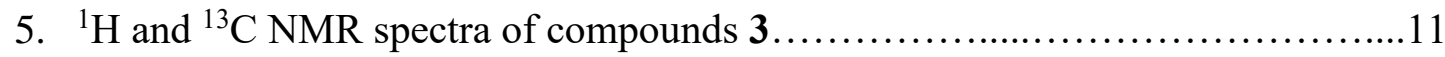

\section{Instrumentation and chemicals}

All reactions involving air- or moisture-sensitive reagents or intermediates were carried out in heat-gun-dried glassware under an argon atmosphere. THF was freshly distilled from potassium under argon. All other solvents and reagents were purified according to standard procedures or were used as received from Aldrich, Acros Organics, Alfa Aesar. The pinacols were synthesized according to literature procedures. ${ }^{1} \mathrm{H}$ NMR and ${ }^{13} \mathrm{C}$ NMR spectra were recorded on a Bruker DPX 300, a Bruker AV 300, a Bruker AV 400 at $300 \mathrm{~K}$. The solvents residual proton resonance and the respective carbon resonance $\left(\mathrm{CHCl}_{3}, \delta=7.26 \mathrm{ppm}\right.$ for ${ }^{1} \mathrm{H}$ NMR, $\delta=77.0 \mathrm{ppm}$ for ${ }^{13} \mathrm{C}$ NMR) was used for calibration. Merck silica gel 60 F 254 plates were used for TLC, detection with UV light and dipping into a solution of $\mathrm{KMnO}_{4}\left(1.5 \mathrm{~g}\right.$ in $400 \mathrm{~mL} \mathrm{H} \mathrm{H}_{2} \mathrm{O}, 5 \mathrm{~g}$ of $\left.\mathrm{NaHCO}_{3}\right)$, followed by heating. Flash column chromatography (FC) was performed using Merck or Fluka silica gel $60(40-63 \mu \mathrm{m})$ with a pressure of 0.3 bar. IR spectra were recorded on a Digilab Varian 3100 FT-IR Excalibur Series. Melting points (M.P.) were determined on a SMP 10 apparatus 
(Stuart Scientific). Mass spectra were recorded on a Bruker MicroTof or an Orbitrap LTQ XL (Nanospray) of Thermo Scientific.

\section{Preparation of pinacols 1}

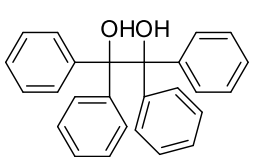

$1 \mathrm{a}$

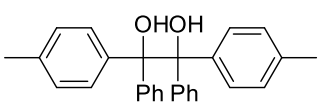

1d

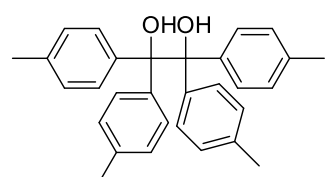

1b

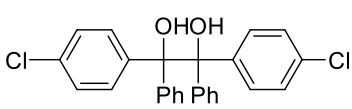

1e

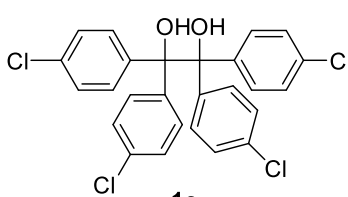

$1 \mathrm{c}$

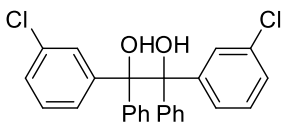

$1 f$

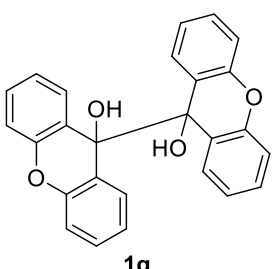

(1b),

1,1,2,2-Tetraphenylethane-1,2-diol

(1a), 1,1,2,2-tetra-p-tolylethane-1,2-diol

1,1,2,2-tetrakis(4-chlorophenyl)ethane-1,2-diol (1c), 1,2-diphenyl-1,2-di-p-tolylethane-1,2-diol $(\mathbf{1 d})$, 1,2-bis(4-chlorophenyl)-1,2-diphenylethane-1,2-diol

(1e),

1,2-bis(3-chlorophenyl)-1,2-diphenylethane-1,2-diol (1f), 9H,9' $H$-[9,9'-bixanthene]-9,9'-diol (1g) were commercially available or prepared according to a literature procedure. ${ }^{1}$

\section{Reaction of iodoarenes with pinacols}

General procedure (GP1): Reaction of iodoarenes with pinacols

$1(0.7 \mathrm{mmol}), \mathrm{NaH}(2.80 \mathrm{mmol})$ and $1 \mathrm{~mL}$ of THF were added sequentially under Ar atmosphere to a flame-dried Schlenk tube equipped with a magnetic stir bar. The mixture was allowed to stir at room temperature for $5 \mathrm{~min}$. After that $2(0.5 \mathrm{mmol})$ and $0.5 \mathrm{~mL}$ of THF were added to the reaction mixture under Ar atmosphere. Then the resulting mixture was stirred at room temperature for $20 \mathrm{~h}$. The reaction was quenched by addition of $5 \mathrm{~mL}$ of saturated aqueous $\mathrm{NH}_{4} \mathrm{Cl}$ solution and the resulting mixture was then extracted with ethyl acetate three times. The combined organic extracts were washed with brine, dried over $\mathrm{Na}_{2} \mathrm{SO}_{4}$, filtered and concentrated. The residual was then purified by flash column chromatography $\left(\mathrm{SiO}_{2}\right)$ to obtain product 3 .

\section{(2-Methoxyphenyl)diphenylmethanol (3aa) ${ }^{2}$}

3aa was prepared according to GP1 with $\mathbf{1 a}(257 \mathrm{mg}, 0.701 \mathrm{mmol}), \mathbf{2 a}(117 \mathrm{mg}, 0.500 \mathrm{mmol})$ and $\mathrm{NaH}(112 \mathrm{mg}, 2.80 \mathrm{mmol})$ in $1.5 \mathrm{~mL}$ of THF at room temperature for $20 \mathrm{~h}$. Purification by flash 
<smiles>COc1ccccc1C(O)(c1ccccc1)c1ccccc1</smiles>
column chromatography (pentane/ethyl acetate $=150 / 1$ to $100 / 1$ ) provided 3aa as a white solid (99 mg, 68\%); MP: $126{ }^{\circ} \mathrm{C} ;{ }^{1} \mathbf{H}$ NMR $\left(300 \mathrm{MHz}, \mathrm{CDCl}_{3}\right) \delta 7.27-$ $7.10(\mathrm{~m}, 11 \mathrm{H}), 6.86(\mathrm{dd}, J=8.2,1.1 \mathrm{~Hz}, 1 \mathrm{H}), 6.73(\mathrm{td}, J=7.6,1.2 \mathrm{~Hz}, 1 \mathrm{H}), 6.44$ $(\mathrm{dd}, J=7.7,1.6 \mathrm{~Hz}, 1 \mathrm{H}), 5.20(\mathrm{~s}, 1 \mathrm{H}), 3.55(\mathrm{~s}, 3 \mathrm{H}) .{ }^{13} \mathbf{C}$ NMR $\left(75 \mathrm{MHz}, \mathrm{CDCl}_{3}\right)$

FTIR (neat): $\tilde{v}=3498,3063,3026,3006,2837,2360,1598,1582,1484,1462,1446,1436,1371$, 1283, 1234, 1160, $1011 \mathrm{~cm}^{-2}$. HRMS (ESI): Exact mass calculated for $\mathrm{C}_{20} \mathrm{H}_{18} \mathrm{O}_{2} \mathrm{Na}\left([\mathrm{M}+\mathrm{Na}]^{+}\right)$: 313.1199, mass found: 313.1198 .

\section{(2-Ethoxyphenyl)diphenylmethanol (3ab)}<smiles>CCOc1ccccc1C(O)(c1ccccc1)c1ccccc1</smiles>

$3 a b$

3ab was prepared according to GP1 with $\mathbf{1 a}(257 \mathrm{mg}, 0.701 \mathrm{mmol}), \mathbf{2 b}(124 \mathrm{mg}$, $0.500 \mathrm{mmol})$ and $\mathrm{NaH}(112 \mathrm{mg}, 2.80 \mathrm{mmol})$ in $1.5 \mathrm{~mL}$ of $\mathrm{THF}$ at room temperature for $20 \mathrm{~h}$. Purification by flash column chromatography (pentane/ethyl acetate $=150 / 1)$ provided 3ab as a white solid $(106 \mathrm{mg}, 70 \%)$; MP: $136{ }^{\circ} \mathrm{C} ;{ }^{1} \mathbf{H}$ NMR $\left(300 \mathrm{MHz}, \mathrm{CDCl}_{3}\right) \delta$ 7.28-7.10 (m, 11H), $6.82(\mathrm{dd}, J=8.2,1.1 \mathrm{~Hz}, 1 \mathrm{H}), 6.72(\mathrm{td}, J=7.5,1.2$ $\mathrm{Hz}, 1 \mathrm{H}), 6.44(\mathrm{dd}, J=7.7,1.7 \mathrm{~Hz}, 1 \mathrm{H}), 5.24(\mathrm{~s}, 1 \mathrm{H}), 3.78(\mathrm{q}, J=7.0 \mathrm{~Hz}, 2 \mathrm{H}), 0.89(\mathrm{t}, J=7.0 \mathrm{~Hz}, 3 \mathrm{H})$. ${ }^{13}$ C NMR $\left(75 \mathrm{MHz}, \mathrm{CDCl}_{3}\right) \delta 156.75,146.64,135.87,129.98,128.81,127.69,127.60,126.85$, 120.35, 113.00, 81.88, 64.41, 14.33. FTIR (neat): $\tilde{v}=3483,3059,2983,1598,1583,1487,1474$, 1445, 1393, 1378, 1362, 1286, 1265, 1234, 1118, 1032, $1011 \mathrm{~cm}^{-2}$. HRMS (ESI): Exact mass calculated for $\mathrm{C}_{21} \mathrm{H}_{20} \mathrm{O}_{2} \mathrm{Na}\left([\mathrm{M}+\mathrm{Na}]^{+}\right)$: 327.1356 , mass found: 327.1368 .

\section{(2-(Methylthio)phenyl)diphenylmethanol (3ac) $)^{3}$}<smiles>Cc1ccccc1C(O)(c1ccccc1)c1ccccc1</smiles>

(pentane/ethyl acetate $=200 / 1$ to $100 / 1)$ provided 3ac as a white solid $(80 \mathrm{mg}$,

52\%); MP: $91{ }^{\circ} \mathrm{C} ;{ }^{1} \mathbf{H}$ NMR $\left(300 \mathrm{MHz}, \mathrm{CDCl}_{3}\right) \delta 7.37(\mathrm{dd}, J=7.8,1.4 \mathrm{~Hz}, 1 \mathrm{H}), 7.29-7.13(\mathrm{~m}$, 11H), $6.95(\mathrm{td}, J=7.6,1.4 \mathrm{~Hz}, 1 \mathrm{H}), 6.52(\mathrm{dd}, J=7.9,1.5 \mathrm{~Hz}, 1 \mathrm{H}), 5.89(\mathrm{~s}, 1 \mathrm{H}), 2.22(\mathrm{~s}, 3 \mathrm{H}) .{ }^{13} \mathrm{C}$ NMR $\left(75 \mathrm{MHz}, \mathrm{CDCl}_{3}\right) \delta 147.07,146.44,136.23,131.83,130.10,128.17,128.02,127.87,127.12$, 125.87, 82.77, 18.71. FTIR (neat): $\tilde{v}=3418,3058,2926,1741,1490,1447,1356,1265,1162$, 
$1016 \mathrm{~cm}^{-2}$. HRMS (ESI): Exact mass calculated for $\mathrm{C}_{20} \mathrm{H}_{18} \mathrm{OSNa}\left([\mathrm{M}+\mathrm{Na}]^{+}\right): 329.0971$, mass found: 329.0974 .

\section{Naphthalen-1-yldiphenylmethanol (3ad)}<smiles>OC(c1ccccc1)(c1ccccc1)c1cccc2ccccc12</smiles>

$3 \mathrm{ad}$

3ad was prepared according to GP1 with 1 a $(257 \mathrm{mg}, 0.701 \mathrm{mmol}), \mathbf{2 d}(127$ $\mathrm{mg}, 0.500 \mathrm{mmol})$ and $\mathrm{NaH}(112 \mathrm{mg}, 2.80 \mathrm{mmol})$ in $1.5 \mathrm{~mL}$ of THF at room temperature for $20 \mathrm{~h}$. Purification by flash column chromatography (pentane/ethyl acetate $=150 / 1$ to $100 / 1)$ provided 3ad as a yellow solid $(77 \mathrm{mg}$, 50\%); MP: $130{ }^{\circ} \mathrm{C} ;{ }^{1} \mathbf{H}$ NMR $\left(300 \mathrm{MHz}, \mathrm{CDCl}_{3}\right) \delta 8.01(\mathrm{~d}, J=8.7 \mathrm{~Hz}, 1 \mathrm{H}), 7.73$ (dd, $J=13.0,8.0 \mathrm{~Hz}, 2 \mathrm{H}), 7.34-7.12(\mathrm{~m}, 13 \mathrm{H}), 6.77(\mathrm{dd}, J=7.3,1.2 \mathrm{~Hz}, 1 \mathrm{H}), 3.22(\mathrm{~s}, 1 \mathrm{H}) .{ }^{13} \mathrm{C}$ NMR $\left(75 \mathrm{MHz}, \mathrm{CDCl}_{3}\right) \delta 146.97,142.09,134.98,131.32,129.33,128.80,128.18,128.05,128.00$, 127.76, 127.14, 125.53, 125.29, 124.22, 83.30. FTIR (neat): $\tilde{v}=3563,3055,1598,1508,1492$, 1446, 1256, 1213, 1156, 1032, $1002 \mathrm{~cm}^{-2}$. HRMS (ESI): Exact mass calculated for $\mathrm{C}_{23} \mathrm{H}_{18} \mathrm{ONa}$ $\left([\mathrm{M}+\mathrm{Na}]^{+}\right): 333.1250$, mass found: 333.1255 .

\section{Furan-2-yldiphenylmethanol (3ae) ${ }^{4}$}

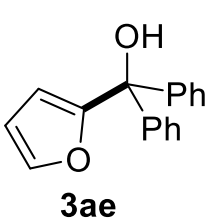

3ae was prepared according to GP1 with $\mathbf{1 a}(257 \mathrm{mg}, 0.701 \mathrm{mmol}), \mathbf{2 e}(97 \mathrm{mg}$, $0.500 \mathrm{mmol})$ and $\mathrm{NaH}(112 \mathrm{mg}, 2.80 \mathrm{mmol})$ in $1.5 \mathrm{~mL}$ of $\mathrm{THF}$ at room temperature for $20 \mathrm{~h}$. Purification by flash column chromatography (pentane/ethyl acetate $=150 / 1$ to $100 / 1)$ provided 3ae as a white solid $(61 \mathrm{mg}$, 49\%); MP: $96{ }^{\circ} \mathrm{C} ;{ }^{1} \mathbf{H}$ NMR (300 MHz, $\left.\mathrm{CDCl}_{3}\right) \delta 7.38-7.33(\mathrm{~m}, 1 \mathrm{H}), 7.25-7.17(\mathrm{~m}, 10 \mathrm{H}), 6.23$ $(\mathrm{dd}, J=3.3,1.9 \mathrm{~Hz}, 1 \mathrm{H}), 5.84(\mathrm{dd}, J=3.2,0.9 \mathrm{~Hz}, 1 \mathrm{H}), 3.01(\mathrm{~s}, 1 \mathrm{H}) .{ }^{13} \mathbf{C} \mathbf{N M R}\left(75 \mathrm{MHz}, \mathrm{CDCl}_{3}\right) \delta$ $157.87,144.62,142.59,127.95,127.61,127.19,110.04,109.58,78.00$. FTIR (neat): $\tilde{v}=3424,3062$, 2926, 1491, 1447, 1338, 1167, 1145, $1011 \mathrm{~cm}^{-2}$. HRMS (ESI): Exact mass calculated for $\mathrm{C}_{17} \mathrm{H}_{14} \mathrm{O}_{2} \mathrm{Na}\left([\mathrm{M}+\mathrm{Na}]^{+}\right)$: 273.0886, mass found: 273.0905 .

\section{Diphenyl(pyridin-2-yl)methanol (3af) ${ }^{5}$}

3af was prepared according to GP1 with $\mathbf{1 a}(257 \mathrm{mg}, 0.701 \mathrm{mmol}), \mathbf{2 f}(103 \mathrm{mg}, 0.502 \mathrm{mmol})$ and $\mathrm{NaH}(112 \mathrm{mg}, 2.80 \mathrm{mmol})$ in $1.5 \mathrm{~mL}$ of THF at room temperature for $20 \mathrm{~h}$. Purification by flash column chromatography (pentane/ethyl acetate $=80 / 1)$ provided 3af as a white solid $(58 \mathrm{mg}$, 
<smiles>OC(c1ccccc1)(c1ccccc1)c1ccccn1</smiles>

3af

44\%); MP: $106{ }^{\circ} \mathrm{C} ;{ }^{1} \mathbf{H}$ NMR $\left(400 \mathrm{MHz}, \mathrm{CDCl}_{3}\right) \delta 8.62(\mathrm{dt}, J=5.0,1.3 \mathrm{~Hz}, 1 \mathrm{H})$, $7.66(\mathrm{td}, J=7.7,1.8 \mathrm{~Hz}, 1 \mathrm{H}), 7.35-7.23(\mathrm{~m}, 10 \mathrm{H}), 7.14(\mathrm{dt}, J=8.0,1.0 \mathrm{~Hz}, 1 \mathrm{H}), 6.33$ $(\mathrm{s}, 1 \mathrm{H}) .{ }^{13} \mathrm{C}$ NMR $\left(100 \mathrm{MHz}, \mathrm{CDCl}_{3}\right) \delta 163.15,147.70,146.08,136.37,128.11$, 127.89, 127.28, 122.88, 122.32, 80.80. FTIR (neat): $\tilde{v}=3338,3059,1591,1572$, 1490, 1447, 1433, 1376, 1265, 1169, $1038 \mathrm{~cm}^{-2}$. HRMS (ESI): Exact mass calculated for $\mathrm{C}_{18} \mathrm{H}_{15} \mathrm{NONa}\left([\mathrm{M}+\mathrm{Na}]^{+}\right): 284.1046$, mass found: 284.1043.

\section{Isoquinolin-1-yldiphenylmethanol (3ag)}<smiles>OC(c1ccccc1)(c1ccccc1)c1nccc2ccccc12</smiles>

3 ag

3ag was prepared according to GP1 with $\mathbf{1 a}(257 \mathrm{mg}, 0.701 \mathrm{mmol}), \mathbf{2 g}(128 \mathrm{mg}$, $0.502 \mathrm{mmol})$ and $\mathrm{NaH}(112 \mathrm{mg}, 2.80 \mathrm{mmol})$ in $1.5 \mathrm{~mL}$ of THF at room temperature for $20 \mathrm{~h}$. Purification by flash column chromatography (pentane/ethyl acetate $=80 / 1$ to $50 / 1$ ) provided 3ag as a white solid (63 $\mathrm{mg}$, 40\%); MP: $143{ }^{\circ} \mathrm{C} ;{ }^{1} \mathbf{H}$ NMR (400 MHz, $\left.\mathrm{CDCl}_{3}\right) \delta 8.43(\mathrm{~d}, J=5.6 \mathrm{~Hz}, 1 \mathrm{H}), 7.92$ (s, 1H), $7.77-7.70(\mathrm{~m}, 1 \mathrm{H}), 7.65-7.56(\mathrm{~m}, 2 \mathrm{H}), 7.46(\mathrm{ddd}, J=8.1,6.8,1.1 \mathrm{~Hz}, 1 \mathrm{H}), 7.31-7.26(\mathrm{~m}$, 4H), $7.21-7.13(\mathrm{~m}, 7 \mathrm{H}) .{ }^{13} \mathbf{C}$ NMR $\left(100 \mathrm{MHz}, \mathrm{CDCl}_{3}\right) \delta 161.43,145.40,139.12,137.52,129.79$, $128.83,128.00,127.90,127.39,127.32,126.67,126.31,121.98,80.73$. FTIR (neat): $\tilde{v}=3246,3056$, 1623, 1589, 1560, 1493, 1444, 1368, 1326, 1163, $1000 \mathrm{~cm}^{-2}$. HRMS (ESI): Exact mass calculated for $\mathrm{C}_{22} \mathrm{H}_{18} \mathrm{NO}\left([\mathrm{M}+\mathrm{H}]^{+}\right): 312.1383$, mass found: 312.1403 .

\section{Diphenyl(thiophen-2-yl)methanol (3ah)}<smiles>OC(c1ccccc1)(c1ccccc1)c1cccs1</smiles>

3ah was prepared according to GP1 with $\mathbf{1 a}(257 \mathrm{mg}, 0.701 \mathrm{mmol}), \mathbf{2 h}(105 \mathrm{mg}$, $0.500 \mathrm{mmol})$ and $\mathrm{NaH}(112 \mathrm{mg}, 2.80 \mathrm{mmol})$ in $1.5 \mathrm{~mL}$ of $\mathrm{THF}$ at room temperature for $20 \mathrm{~h}$. Purification by flash column chromatography (pentane/ethyl acetate $=100 / 1)$ provided $3 \mathbf{a h}$ as a white solid $(110 \mathrm{mg}, 83 \%)$; MP: $131{ }^{\circ} \mathrm{C} ;{ }^{1} \mathbf{H}$ NMR $\left(400 \mathrm{MHz}, \mathrm{CDCl}_{3}\right) \delta 7.32-7.17(\mathrm{~m}, 11 \mathrm{H}), 6.86(\mathrm{dd}, J=5.1,3.6 \mathrm{~Hz}, 1 \mathrm{H})$, $6.64(\mathrm{dd}, J=3.6,1.2 \mathrm{~Hz}, 1 \mathrm{H}), 2.88(\mathrm{~d}, J=1.5 \mathrm{~Hz}, 1 \mathrm{H}) .{ }^{13} \mathbf{C}$ NMR $\left(101 \mathrm{MHz}, \mathrm{CDCl}_{3}\right) \delta 152.18$, 146.50, 127.94, 127.58, 127.21, 126.87, 126.42, 125.62, 80.04. FTIR (neat): $\tilde{v}=3456,3060,1491$, 1447, 1265, 1032, $1006 \mathrm{~cm}^{-2}$. HRMS (ESI): Exact mass calculated for $\mathrm{C}_{17} \mathrm{H}_{14} \mathrm{OSNa}\left([\mathrm{M}+\mathrm{Na}]^{+}\right)$: 289.0658, mass found: 289.0679 . 


\section{Benzo $[b]$ thiophen-2-yldiphenylmethanol (3ai) ${ }^{6}$}

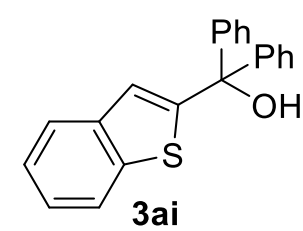

3ai was prepared according to GP1 with $\mathbf{1 a}(257 \mathrm{mg}, 0.701 \mathrm{mmol}), \mathbf{2 i}$ (155 $\mathrm{mg}, 0.500 \mathrm{mmol})$ and $\mathrm{NaH}(112 \mathrm{mg}, 2.80 \mathrm{mmol})$ in $1.5 \mathrm{~mL}$ of THF at room temperature for $20 \mathrm{~h}$. Purification by flash column chromatography (pentane/ethyl acetate $=80 / 1$ to $60 / 1)$ provided 3ai as a white solid (100 mg,

63\%); MP: $80{ }^{\circ} \mathrm{C} ;{ }^{1} \mathbf{H}$ NMR (300 MHz, $\left.\mathrm{CDCl}_{3}\right) \delta 7.71-7.66(\mathrm{~m}, 1 \mathrm{H}), 7.59-7.54(\mathrm{~m}, 1 \mathrm{H}), 7.37-$ $7.31(\mathrm{~m}, 4 \mathrm{H}), 7.30-7.19(\mathrm{~m}, 8 \mathrm{H}), 6.85(\mathrm{~s}, 1 \mathrm{H}), 2.96(\mathrm{~s}, 1 \mathrm{H}) .{ }^{13} \mathbf{C}$ NMR $\left(75 \mathrm{MHz}, \mathrm{CDCl}_{3}\right) \delta 152.68$, $145.86,140.17,139.26,128.06,127.80,127.35,124.36,124.27,123.73,123.57,122.27,80.47$. FTIR (neat): $\tilde{v}=3547,3058,1599,1491,1447,1330,1264,1157,1102,1007 \mathrm{~cm}^{-2}$. HRMS (EI): Exact mass calculated for $\mathrm{C}_{21} \mathrm{H}_{16} \mathrm{OSNa}\left([\mathrm{M}+\mathrm{Na}]^{+}\right)$: 339.0814 , mass found: 339.0810 .

\section{Dibenzo $[b, d]$ thiophen-4-yldiphenylmethanol (3aj)}<smiles>OC(c1ccccc1)(c1ccccc1)c1cccc2c1sc1ccccc12</smiles>

3aj 3aj was prepared according to GP1 with $\mathbf{1 a}(257 \mathrm{mg}, 0.701 \mathrm{mmol}), \mathbf{2} \mathbf{j}(155 \mathrm{mg}$, $0.500 \mathrm{mmol})$ and $\mathrm{NaH}(112 \mathrm{mg}, 2.80 \mathrm{mmol})$ in $1.5 \mathrm{~mL}$ of $\mathrm{THF}$ at room temperature for $20 \mathrm{~h}$. Purification by flash column chromatography (pentane/ethyl acetate $=80 / 1$ to $60 / 1)$ provided 3aj as a white solid (142 mg, 77\%); MP: $216{ }^{\circ} \mathrm{C} ;{ }^{1} \mathbf{H}$ NMR (300 MHz, $\left.\mathrm{CDCl}_{3}\right) \delta 8.07-7.99$ (m, 2H), $7.66-$ $7.59(\mathrm{~m}, 1 \mathrm{H}), 7.35-7.20(\mathrm{~m}, 13 \mathrm{H}), 6.88(\mathrm{dd}, J=7.5,1.1 \mathrm{~Hz}, 1 \mathrm{H}), 3.17(\mathrm{~s}, 1 \mathrm{H}) .{ }^{13} \mathrm{C}$ NMR $\left(75 \mathrm{MHz}, \mathrm{CDCl}_{3}\right) \delta 145.02,141.12,140.67,138.26,136.94,134.81,128.08,128.00,127.65$, 127.42, 126.67, 124.06, 123.79, 122.23, 121.39, 121.02, 82.96. FTIR (neat): $\tilde{v}=3485,3059,1704$, 1492, 1443, 1389, 1160, $1019 \mathrm{~cm}^{-2}$. HRMS (EI): Exact mass calculated for $\mathrm{C}_{25} \mathrm{H}_{18} \mathrm{OS}\left(\mathrm{M}^{+}\right)$: 366.10729, mass found: 366.10678 .

\section{(2-Methoxyphenyl)di-p-tolylmethanol (3ba) ${ }^{7}$}

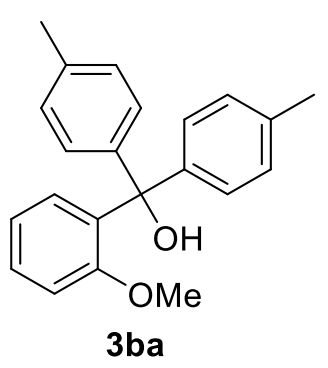

3ba was prepared according to GP1 with $\mathbf{1 b}(296 \mathrm{mg}, 0.700 \mathrm{mmol}), \mathbf{2 a}$ (117 mg, $0.500 \mathrm{mmol})$ and $\mathrm{NaH}(112 \mathrm{mg}, 2.80 \mathrm{mmol})$ in $1.5 \mathrm{~mL}$ of THF at room temperature for $20 \mathrm{~h}$. Purification by flash column chromatography (pentane/ethyl acetate $=100 / 1$ to $80 / 1)$ provided $\mathbf{3 b a}$ as a white solid $(92$ mg, 58\%); MP: $130{ }^{\circ} \mathrm{C} ;{ }^{1} \mathbf{H}$ NMR $\left(300 \mathrm{MHz}, \mathrm{CDCl}_{3}\right) \delta 7.32(\mathrm{ddd}, J=8.2$,

7.4, $1.7 \mathrm{~Hz}, 1 \mathrm{H}), 7.21-7.11(\mathrm{~m}, 8 \mathrm{H}), 6.99(\mathrm{dd}, J=8.2,1.2 \mathrm{~Hz}, 1 \mathrm{H}), 6.87(\mathrm{td}, J=7.5,1.2 \mathrm{~Hz}, 1 \mathrm{H}), 6.60$ 
$(\mathrm{dd}, J=7.7,1.7 \mathrm{~Hz}, 1 \mathrm{H}), 5.30(\mathrm{~s}, 1 \mathrm{H}), 3.71(\mathrm{~s}, 3 \mathrm{H}), 2.39(\mathrm{~s}, 6 \mathrm{H}) .{ }^{13} \mathbf{C}$ NMR $\left(75 \mathrm{MHz}, \mathrm{CDCl}_{3}\right) \delta$ 157.42, 143.93, 136.35, 135.58, 130.23, 128.74, 128.29, 127.69, 120.44, 112.03, 81.82, 55.71, 21.03. FTIR (neat): $\tilde{v}=3525,3024,2922,1583,1509,1485,1462,1436,1361,1285,1234,1019 \mathrm{~cm}^{-2}$. HRMS (ESI): Exact mass calculated for $\mathrm{C}_{22} \mathrm{H}_{22} \mathrm{O}_{2} \mathrm{Na}\left([\mathrm{M}+\mathrm{Na}]^{+}\right)$: 341.1512, mass found: 341.1514 .

\section{Bis(4-chlorophenyl)(2-methoxyphenyl)methanol (3ca)}

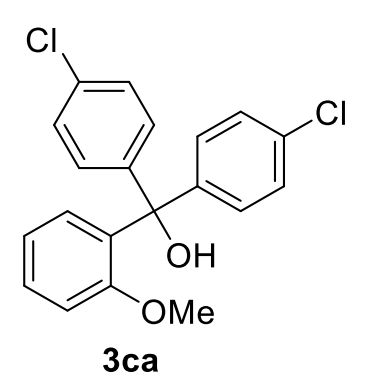

3ca was prepared according to GP1 with $\mathbf{1 c}(353 \mathrm{mg}, 0.700 \mathrm{mmol}), \mathbf{2 a}$ (117 mg, $0.500 \mathrm{mmol})$ and $\mathrm{NaH}(112 \mathrm{mg}, 2.80 \mathrm{mmol})$ in $1.5 \mathrm{~mL}$ of THF at room temperature for $20 \mathrm{~h}$. Purification by flash column chromatography (pentane/ethyl acetate $=120 / 1$ to $80 / 1$ ) provided $\mathbf{3 c a}$ as a white solid (116 mg, 65\%); MP: $108{ }^{\circ} \mathrm{C} ;{ }^{1} \mathbf{H}$ NMR (300 MHz, $\mathrm{CDCl}_{3}$ ) $\delta 7.26-7.15(\mathrm{~m}, 5 \mathrm{H}), 7.11-7.05(\mathrm{~m}, 4 \mathrm{H}), 6.88(\mathrm{dd}, J=8.3,1.2 \mathrm{~Hz}, 1 \mathrm{H})$, $6.76(\operatorname{td}, J=7.6,1.2 \mathrm{~Hz}, 1 \mathrm{H}), 6.41(\mathrm{dd}, J=7.7,1.7 \mathrm{~Hz}, 1 \mathrm{H}), 5.18(\mathrm{~s}, 1 \mathrm{H}), 3.60(\mathrm{~s}, 3 \mathrm{H}) .{ }^{13} \mathbf{C}$ NMR $(75$ $\left.\mathrm{MHz}, \mathrm{CDCl}_{3}\right) \delta 157.12,144.85,134.25,133.07,129.90,129.36,129.14,127.91,120.67,112.04,81.25$, 55.66. FTIR (neat): $\tilde{v}=3520,2974,1584,1485,1437,1400,1265,1235,1091,1013 \mathrm{~cm}^{-2}$. HRMS (ESI): Exact mass calculated for $\mathrm{C}_{20} \mathrm{H}_{16} \mathrm{O}_{2} \mathrm{Cl}_{2} \mathrm{Na}\left([\mathrm{M}+\mathrm{Na}]^{+}\right)$: 381.0420 , mass found: 381.0424 .

\section{(2-Methoxyphenyl)(phenyl)(p-tolyl)methanol (3da)}

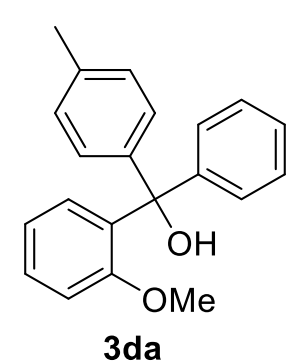

3da was prepared according to GP1 with $\mathbf{1 d}(276 \mathrm{mg}, 0.700 \mathrm{mmol})$, 2a (117 $\mathrm{mg}, 0.500 \mathrm{mmol})$ and $\mathrm{NaH}(112 \mathrm{mg}, 2.80 \mathrm{mmol})$ in $1.5 \mathrm{~mL}$ of THF at room temperature for $20 \mathrm{~h}$. Purification by flash column chromatography (pentane/ethyl acetate $=150 / 1$ to $100 / 1)$ provided 3da as a white solid (102 mg, 67\%); MP: $127{ }^{\circ} \mathrm{C} ;{ }^{1} \mathbf{H}$ NMR (300 MHz, $\left.\mathrm{CDCl}_{3}\right) \delta 7.23-7.12(\mathrm{~m}, 6 \mathrm{H})$, $7.07-6.98(\mathrm{~m}, 4 \mathrm{H}), 6.86$ (d, $J=8.2 \mathrm{~Hz}, 1 \mathrm{H}), 6.73$ (tt, $J=7.5,1.0 \mathrm{~Hz}, 1 \mathrm{H}), 6.45$ (dt, $J=7.7,1.5 \mathrm{~Hz}$, 1H), $5.18(\mathrm{~d}, J=1.1 \mathrm{~Hz}, 1 \mathrm{H}), 3.56(\mathrm{~s}, 3 \mathrm{H}), 2.25$ (s, 3H). ${ }^{13} \mathbf{C} \mathbf{N M R}\left(75 \mathrm{MHz}, \mathrm{CDCl}_{3}\right) \delta$ 157.39, $146.92,143.65,136.46,135.49,130.22,128.82,128.34,127.73,127.59,126.85,120.48,112.07,81.91$, 55.71, 21.03. FTIR (neat): $\tilde{v}=3521,3025,2839,1598,1583,1487,1464,1448,1435,1361,1287$, 1238, 1183, $1111 \mathrm{~cm}^{-2}$. HRMS (ESI): Exact mass calculated for $\mathrm{C}_{21} \mathrm{H}_{20} \mathrm{O}_{2} \mathrm{Na}\left([\mathrm{M}+\mathrm{Na}]^{+}\right)$: 


\section{(4-Chlorophenyl)(2-methoxyphenyl)(phenyl)methanol (3ea)}

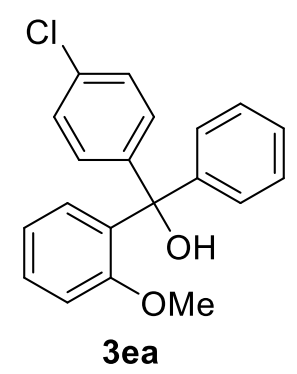

3ea was prepared according to GP1 with $\mathbf{1 e}$ (305 mg, $0.701 \mathrm{mmol})$, 2a (117 $\mathrm{mg}, 0.500 \mathrm{mmol})$ and $\mathrm{NaH}(112 \mathrm{mg}, 2.80 \mathrm{mmol})$ in $1.5 \mathrm{~mL}$ of THF at room temperature for $20 \mathrm{~h}$. Purification by flash column chromatography (pentane/ethyl acetate $=100 / 1$ to $80 / 1)$ provided 3ea as a white solid (98 mg, 60\%); MP: $113{ }^{\circ} \mathrm{C} ;{ }^{1} \mathbf{H}$ NMR (300 MHz, $\left.\mathrm{CDCl}_{3}\right) \delta 7.26-7.07$ (m, 10H), 6.87 $(\mathrm{dd}, J=8.2,1.1 \mathrm{~Hz}, 1 \mathrm{H}), 6.74(\mathrm{td}, J=7.5,1.1 \mathrm{~Hz}, 1 \mathrm{H}), 6.42(\mathrm{dt}, J=7.7,1.4 \mathrm{~Hz}$, 1H), $5.21-5.15(\mathrm{~m}, 1 \mathrm{H}), 3.58(\mathrm{~s}, 3 \mathrm{H}) .{ }^{13} \mathbf{C}$ NMR $\left(75 \mathrm{MHz}, \mathrm{CDCl}_{3}\right) \delta 157.25,146.14,145.37,134.83$, 132.83, 130.06, 129.24, 129.14, 127.78, 127.76, 127.70, 127.16, 120.60, 112.07, 81.63, 55.68. FTIR (neat): $\tilde{v}=3529,3059,1599,1584,1485,1462,1436,1399,1357,1285,1233,1180,1091,1013$ $\mathrm{cm}^{-2}$. HRMS (ESI): Exact mass calculated for $\mathrm{C}_{20} \mathrm{H}_{17} \mathrm{O}_{2} \mathrm{ClNa}\left([\mathrm{M}+\mathrm{Na}]^{+}\right): 347.0809$, mass found: 347.0817 .

\section{(3-Chlorophenyl)(2-methoxyphenyl)(phenyl)methanol (3fa)}

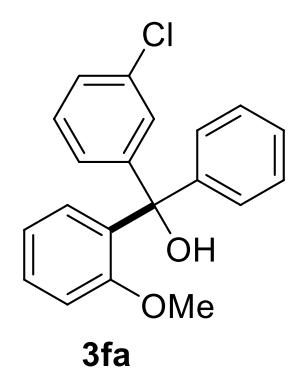

3fa was prepared according to GP1 with $\mathbf{1 f}$ (305 mg, $0.701 \mathrm{mmol})$, 2a (117 $\mathrm{mg}, 0.500 \mathrm{mmol})$ and $\mathrm{NaH}(112 \mathrm{mg}, 2.80 \mathrm{mmol})$ in $1.5 \mathrm{~mL}$ of THF at room temperature for $20 \mathrm{~h}$. Purification by flash column chromatography (pentane/ethyl acetate $=150 / 1$ to $100 / 1)$ provided 3fa as a white solid (109 mg, 67\%); MP: $134{ }^{\circ} \mathrm{C} ;{ }^{1} \mathbf{H}$ NMR (300 MHz, $\left.\mathrm{CDCl}_{3}\right) \delta 7.28-7.07$ (m, 9H), $6.99(\mathrm{dt}, J=7.2,1.7 \mathrm{~Hz}, 1 \mathrm{H}), 6.87(\mathrm{dd}, J=8.2,1.1 \mathrm{~Hz}, 1 \mathrm{H}), 6.75(\mathrm{td}, J=7.6,1.1$

$\mathrm{Hz}, 1 \mathrm{H}), 6.43(\mathrm{dd}, J=7.7,1.7 \mathrm{~Hz}, 1 \mathrm{H}), 5.20(\mathrm{~s}, 1 \mathrm{H}), 3.57(\mathrm{~s}, 3 \mathrm{H}) .{ }^{13} \mathbf{C} \mathbf{N M R}\left(75 \mathrm{MHz}, \mathrm{CDCl}_{3}\right) \delta$ $157.21,148.97,145.86,134.65,133.88,130.06,129.19,128.81,127.90,127.78,127.70,127.21$, 127.17, 126.04, 120.63, 112.10, 81.64, 55.68. FTIR (neat): $\tilde{v}=3516,3063,1593,1486,1463,1357$, 1285, 1233, 1180, 1163, 1116, $1020 \mathrm{~cm}^{-2}$. HRMS (ESI): Exact mass calculated for $\mathrm{C}_{20} \mathrm{H}_{17} \mathrm{O}_{2} \mathrm{ClNa}$ $\left([\mathrm{M}+\mathrm{Na}]^{+}\right): 347.0809$, mass found: 347.0840 . 


\section{9-(2-Methoxyphenyl)-9H-xanthen-9-ol (3ga)}

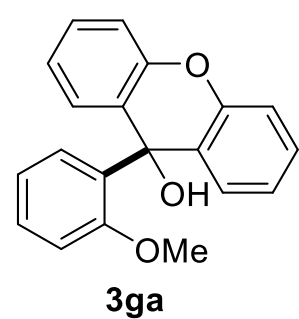

3ga was prepared according to GP1 with $\mathbf{1 g}(276 \mathrm{mg}, 0.700 \mathrm{mmol}), \mathbf{2 a}(117$ $\mathrm{mg}, 0.500 \mathrm{mmol})$ and $\mathrm{NaH}(112 \mathrm{mg}, 2.80 \mathrm{mmol})$ in $1.5 \mathrm{~mL}$ of THF at room temperature for $20 \mathrm{~h}$. Purification by flash column chromatography (pentane/ethyl acetate $=150 / 1$ to $100 / 1)$ provided 3ga as a white solid (59 mg, 39\%); MP: $169{ }^{\circ} \mathrm{C} ;{ }^{1} \mathbf{H}$ NMR $\left(300 \mathrm{MHz}, \mathrm{CDCl}_{3}\right) \delta 7.88(\mathrm{dd}, J=7.7,1.8$ $\mathrm{Hz}, 1 \mathrm{H}), 7.22-7.11(\mathrm{~m}, 5 \mathrm{H}), 7.09-7.02(\mathrm{~m}, 2 \mathrm{H}), 6.97(\mathrm{td}, J=7.6,1.2 \mathrm{~Hz}, 1 \mathrm{H}), 6.93-6.85(\mathrm{~m}, 2 \mathrm{H})$, $6.62(\mathrm{dd}, J=8.1,1.2 \mathrm{~Hz}, 1 \mathrm{H}), 3.18(\mathrm{~s}, 3 \mathrm{H}), 2.99(\mathrm{~s}, 1 \mathrm{H}) .{ }^{13} \mathbf{C}$ NMR $\left(75 \mathrm{MHz}, \mathrm{CDCl}_{3}\right) \delta 150.00$, $128.82,128.55,128.06,127.00,125.88,122.77,120.35,115.74,112.71,55.58$. FTIR (neat): $\tilde{v}=$ $3501,3418,3048,1604,1576,1479,1460,1447,1317,1290,1246,1178,1151,1129,1021 \mathrm{~cm}^{-2}$. HRMS (ESI): Exact mass calculated for $\mathrm{C}_{20} \mathrm{H}_{16} \mathrm{O}_{3} \mathrm{Na}\left([\mathrm{M}+\mathrm{Na}]^{+}\right)$: 327.0992, mass found: 327.1015 .

\section{(2-(Allyloxy)phenyl)diphenylmethanol (3ak)}

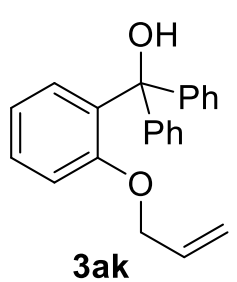

3ak was prepared according to GP1 with $\mathbf{1 a}(257 \mathrm{mg}, 0.701 \mathrm{mmol}), \mathbf{2 k}(130$ $\mathrm{mg}, 0.500 \mathrm{mmol})$ and $\mathrm{NaH}(112 \mathrm{mg}, 2.80 \mathrm{mmol})$ in $1.5 \mathrm{~mL}$ of THF at room temperature for $20 \mathrm{~h}$. Purification by flash column chromatography (pentane/ethyl acetate $=150 / 1)$ provided 3ak as a white solid $(98 \mathrm{mg}, 62 \%)$; MP: $104{ }^{\circ} \mathrm{C} ;{ }^{1} \mathbf{H}$ NMR $\left(300 \mathrm{MHz}, \mathrm{CDCl}_{3}\right) \delta 7.27-7.10(\mathrm{~m}, 11 \mathrm{H}), 6.84(\mathrm{dd}, J=$ 8.2, $1.2 \mathrm{~Hz}, 1 \mathrm{H}), 6.74(\mathrm{td}, J=7.6,1.2 \mathrm{~Hz}, 1 \mathrm{H}), 6.46(\mathrm{dd}, J=7.7,1.7 \mathrm{~Hz}, 1 \mathrm{H}), 5.58-5.41(\mathrm{~m}, 1 \mathrm{H})$, $5.17(\mathrm{~s}, 1 \mathrm{H}), 5.03-4.89(\mathrm{~m}, 2 \mathrm{H}), 4.27(\mathrm{dq}, J=5.1,1.7 \mathrm{~Hz}, 2 \mathrm{H}) .{ }^{13} \mathbf{C} \mathbf{N M R}\left(75 \mathrm{MHz}, \mathrm{CDCl}_{3}\right) \delta$ $156.42,146.58,135.97,132.20,130.15,128.84,127.74,127.65,126.93,120.68,117.73,113.33,81.90$, 69.44. FTIR (neat): $\tilde{v}=3514,3061,1598,1583,1484,1446,1356,1285,1229,1115,1017 \mathrm{~cm}^{-2}$. HRMS (ESI): Exact mass calculated for $\mathrm{C}_{22} \mathrm{H}_{20} \mathrm{O}_{2} \mathrm{Na}\left([\mathrm{M}+\mathrm{Na}]^{+}\right)$: 339.1356, mass found: 339.1356

\section{(2-(But-3-en-2-yloxy)phenyl)diphenylmethanol (3al)}

3al was prepared according to GP1 with 1a $(257 \mathrm{mg}, 0.701 \mathrm{mmol}), 21(137 \mathrm{mg}, 0.500 \mathrm{mmol})$ and $\mathrm{NaH}(112 \mathrm{mg}, 2.80 \mathrm{mmol})$ in $1.5 \mathrm{~mL}$ of THF at room temperature for $20 \mathrm{~h}$. Purification by flash column chromatography (pentane/ethyl acetate $=150 / 1)$ provided 3al as a white solid (94 mg, 
<smiles>C=CC(C)Oc1ccccc1C(O)(c1ccccc1)c1ccccc1</smiles>

57\%); MP: $110{ }^{\circ} \mathrm{C} ;{ }^{1} \mathbf{H}$ NMR (300 MHz, $\left.\mathrm{CDCl}_{3}\right) \delta 7.26-7.14(\mathrm{~m}, 11 \mathrm{H}), 6.85(\mathrm{~d}$, $J=8.1 \mathrm{~Hz}, 1 \mathrm{H}), 6.74(\mathrm{t}, J=7.5 \mathrm{~Hz}, 1 \mathrm{H}), 6.44(\mathrm{dt}, J=7.7,1.9 \mathrm{~Hz}, 1 \mathrm{H}), 5.46-5.33$ (m, 1H), $5.27(\mathrm{~s}, 1 \mathrm{H}), 5.09(\mathrm{dtt}, J=15.2,5.9,1.6 \mathrm{~Hz}, 1 \mathrm{H}), 4.27-4.15(\mathrm{~m}, 2 \mathrm{H})$, $1.52(\mathrm{~d}, J=6.5 \mathrm{~Hz}, 3 \mathrm{H}) .{ }^{13} \mathrm{C} \mathbf{N M R}\left(75 \mathrm{MHz}, \mathrm{CDCl}_{3}\right) \delta 156.58,146.59,135.99$, $130.49,130.04,128.78,127.74,127.60,126.87,124.98,120.45,113.49,81.88$ 69.43, 17.73. FTIR (neat): $\tilde{v}=3512,3060,1598,1583,1482,1447,1377,1358,1283,1226,1111$, $1016 \mathrm{~cm}^{-2}$. HRMS (ESI): Exact mass calculated for $\mathrm{C}_{23} \mathrm{H}_{22} \mathrm{O}_{2} \mathrm{Na}\left([\mathrm{M}+\mathrm{Na}]^{+}\right): 353.1512$, mass found: 353.1513 .

\section{(2-((2-Methylallyl)oxy)phenyl)diphenylmethanol (3am)}

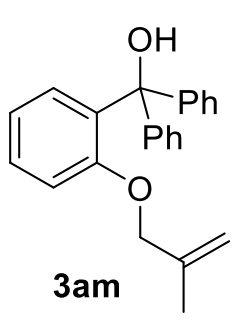

3am was prepared according to GP1 with $\mathbf{1 a}(257 \mathrm{mg}, 0.701 \mathrm{mmol}), \mathbf{2 m}(137$ $\mathrm{mg}, 0.500 \mathrm{mmol})$ and $\mathrm{NaH}(112 \mathrm{mg}, 2.80 \mathrm{mmol})$ in $1.5 \mathrm{~mL}$ of THF at room temperature for $20 \mathrm{~h}$. Purification by flash column chromatography (pentane/ethyl acetate $=200 / 1)$ provided 3am as a white solid $(91 \mathrm{mg}, 55 \%)$; MP: $96{ }^{\circ} \mathrm{C} ;{ }^{1} \mathbf{H}$ NMR $\left(300 \mathrm{MHz}, \mathrm{CDCl}_{3}\right) \delta 7.25-7.14(\mathrm{~m}, 11 \mathrm{H}), 6.85(\mathrm{dd}, J=8.3$, $1.0 \mathrm{~Hz}, 1 \mathrm{H}), 6.74(\mathrm{td}, J=7.6,1.1 \mathrm{~Hz}, 1 \mathrm{H}), 6.44(\mathrm{dt}, J=7.6,1.3 \mathrm{~Hz}, 1 \mathrm{H}), 5.23(\mathrm{~s}, 1 \mathrm{H}), 4.71(\mathrm{~d}, J=16.7$ $\mathrm{Hz}, 2 \mathrm{H}), 4.20(\mathrm{~s}, 2 \mathrm{H}), 1.32(\mathrm{~s}, 3 \mathrm{H}) .{ }^{13} \mathrm{C}$ NMR $\left(75 \mathrm{MHz}, \mathrm{CDCl}_{3}\right) \delta 156.29,146.54,139.97,135.34$, $130.20,128.86,127.74,127.68,126.95,120.46,113.32,112.49,81.91,72.21,18.89$. FTIR (neat): $\tilde{v}=$ 3526, 3036, 1598, 1584, 1484, 1447, 1265, 1227, $1014 \mathrm{~cm}^{-2}$. HRMS (ESI): Exact mass calculated for $\mathrm{C}_{23} \mathrm{H}_{22} \mathrm{O}_{2} \mathrm{Na}\left([\mathrm{M}+\mathrm{Na}]^{+}\right)$: 353.1512 , mass found: 353.1519 .

\section{References:}

1. Gomberg, M.; Bachmann, W. E. J. Am. Chem. Soc. 1927, 49, 236.

2. Dayaker, G.; Chevallier, F.; Gros, P.; Mongin, F. Tetrahedron 2010, 66, 8904.

3. Schöler, S.; Merz, K.; Puls, A.; Winter, M.; Dyker, G. E. J. Inorg. Chem. 2015, 53.

4. Mongin, F.; Bucher, A.; Bazureau, J.; Bayh, O.; Awad, H.; Trécourt, F. Tetrahedron Lett. 2005, 46, 7989

5. Yang, J.; Dudley, G. J. Org. Chem. 2009, 74, 7998.

6. Bíró, A.; Kotschy, A. Eur. J. Org. Chem. 2007, 1364.

7. Niyomura, O.; Nakaiida, S.; Yamada, R.; Kato, S.; Ishida, M.; Ebihara, M.; Ando, F.; Koketsu, J. Molecules 2009, 14, 2555. 


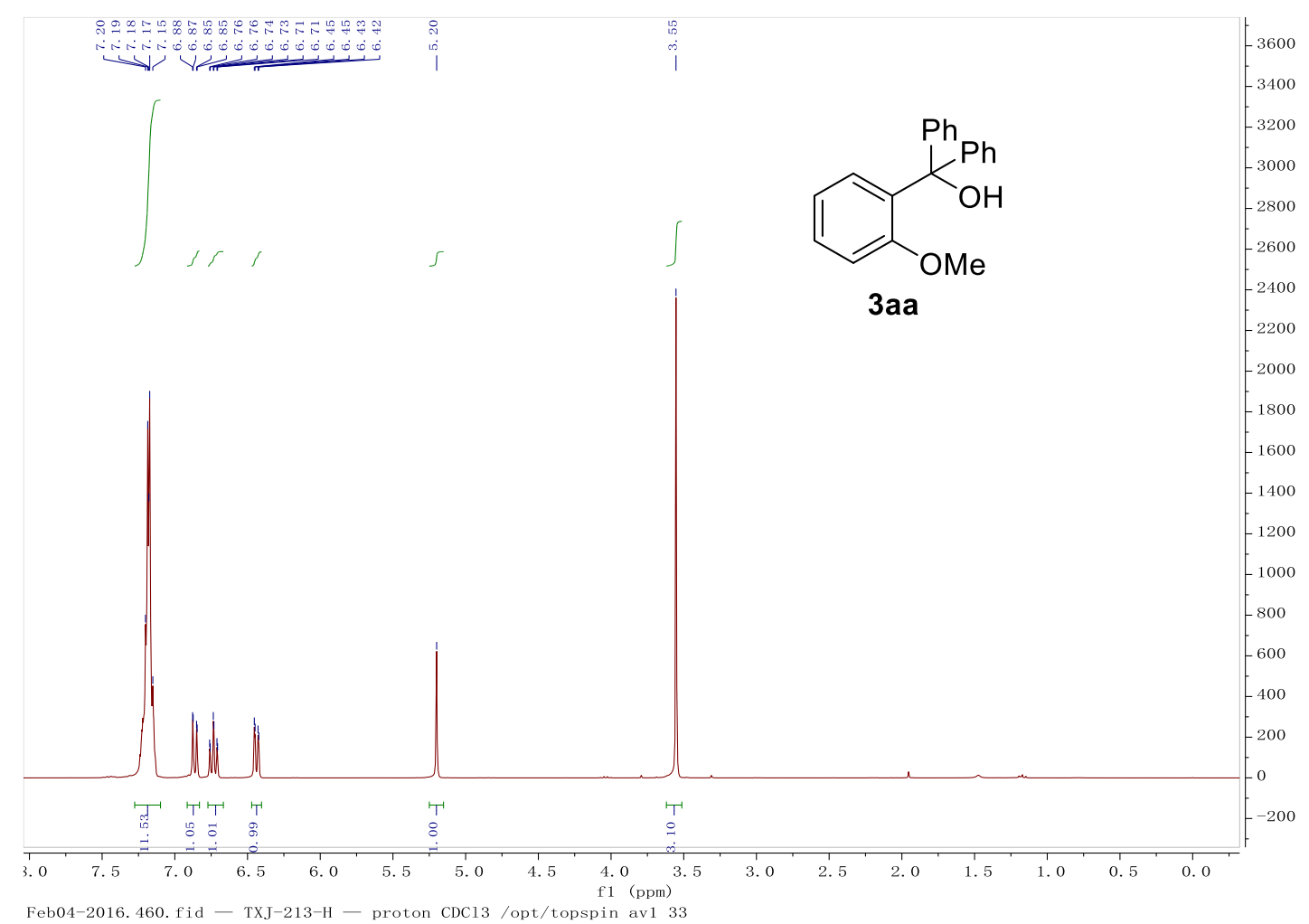

Feb04-2016. 460. fid - TXJ-213-H - proton CDC13/opt/topspin av1 33

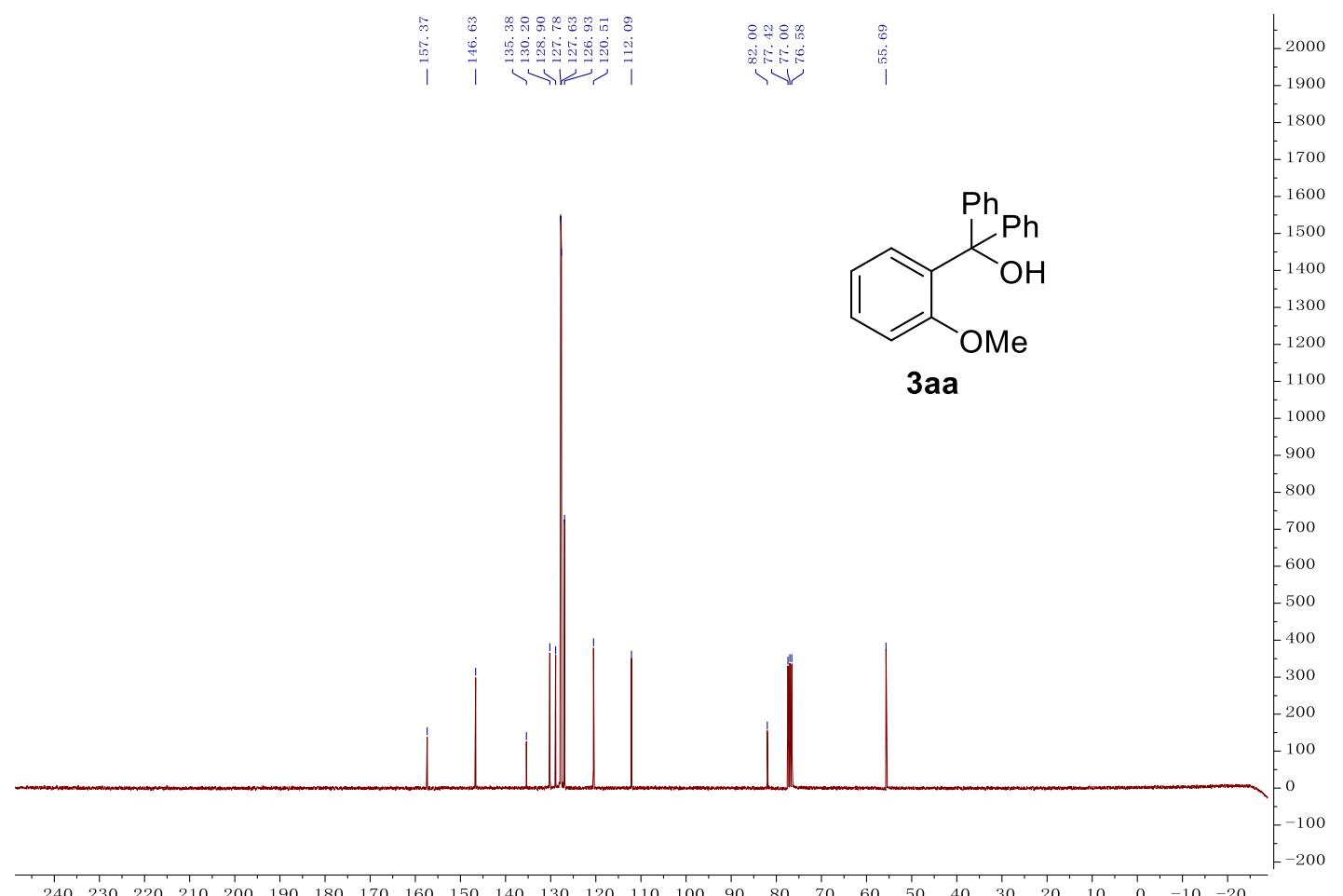

$\begin{array}{llllllllllllllllllllllllllllllll}240 & 230 & 220 & 210 & 200 & 190 & 180 & 170 & 160 & 150 & 140 & 130 & 120 & 110 & 100 & 90 & 80 & 70 & 60 & 50 & 40 & 30 & 20 & 10 & 0 & -10 & -20\end{array}$ Feb04-2016. 461. fid - TXJ-213-C - carbon CDC13 /opt/topspin av1 33 


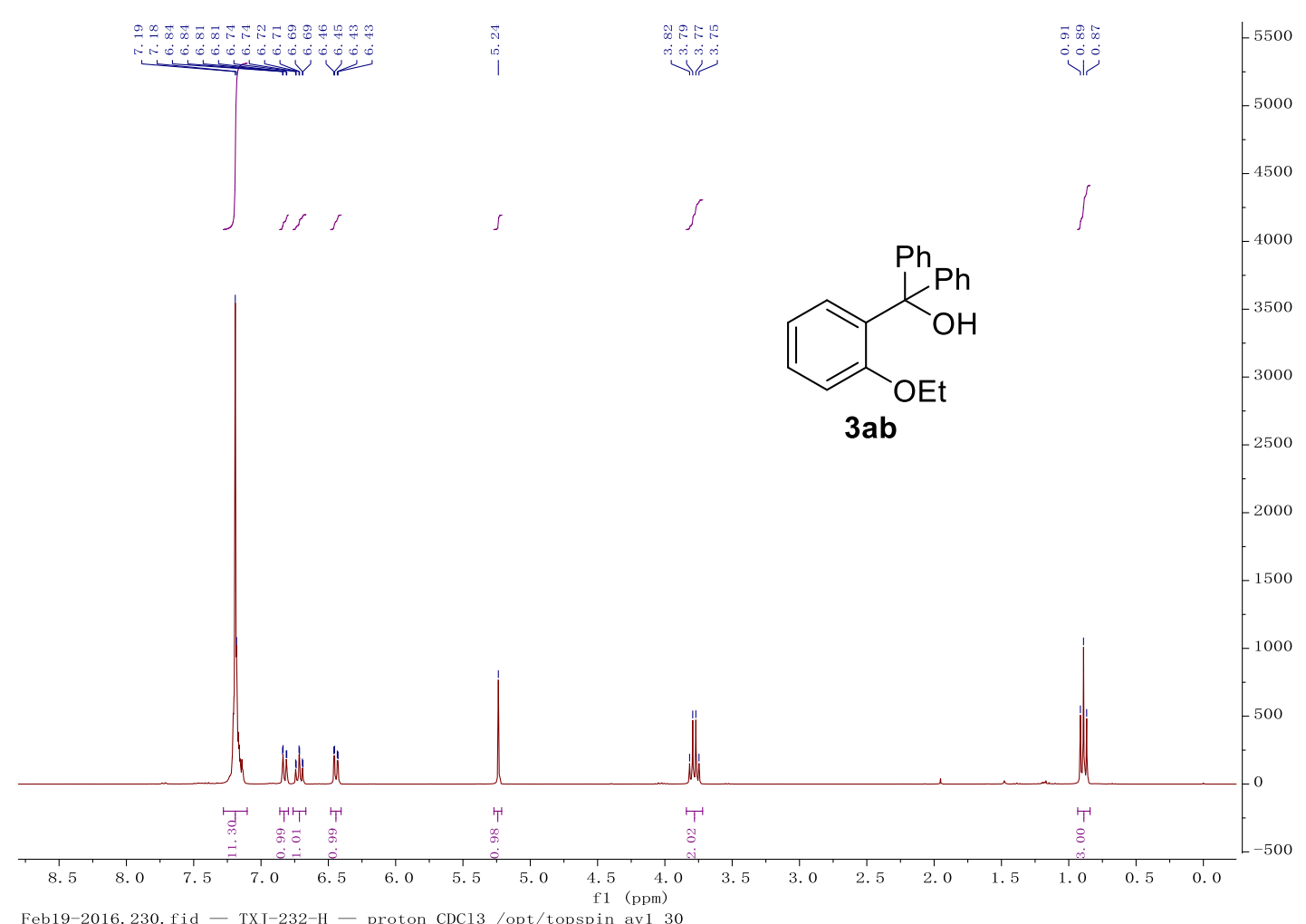

Feb19-2016. 230. fid - TXJ-232-H - proton CDC13/opt/topspin av1 30

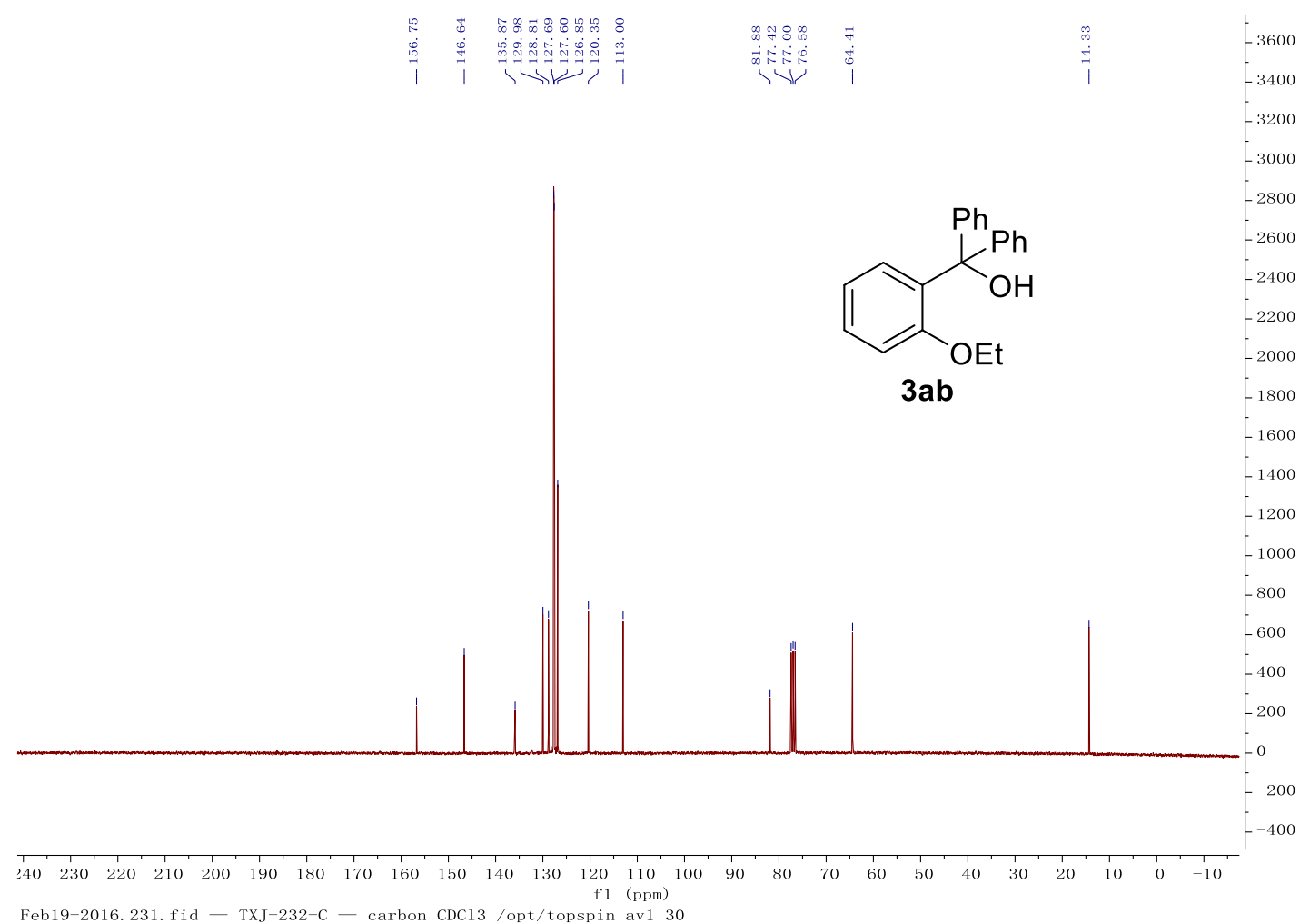

Feb19-2016. 231. fid - TXJ-232-C - carbon CDC13 /opt/topspin av1 30 


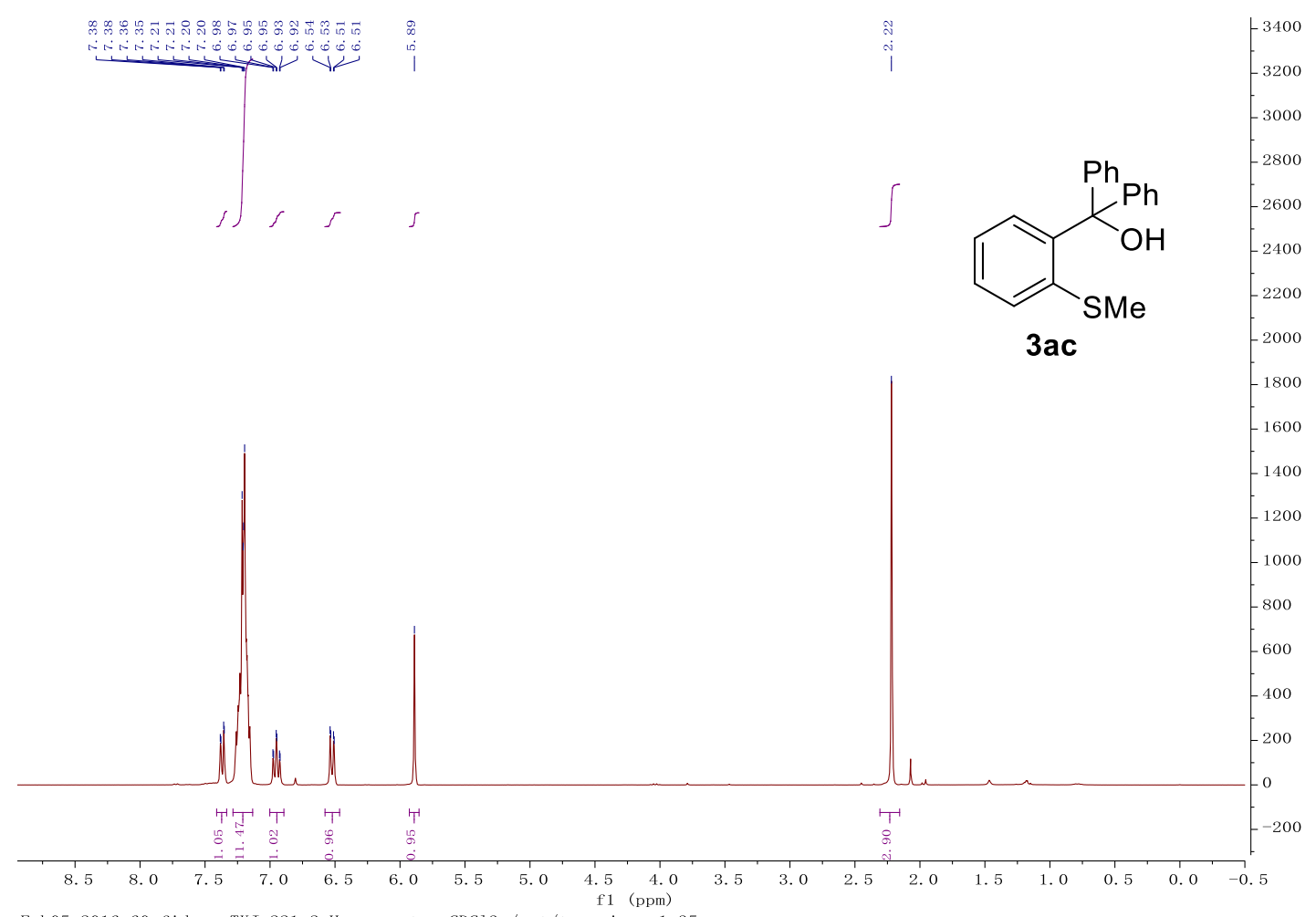

Feb05-2016.60.fid - TXJ-221-2-H - proton CDC13/opt/topspin av1 35

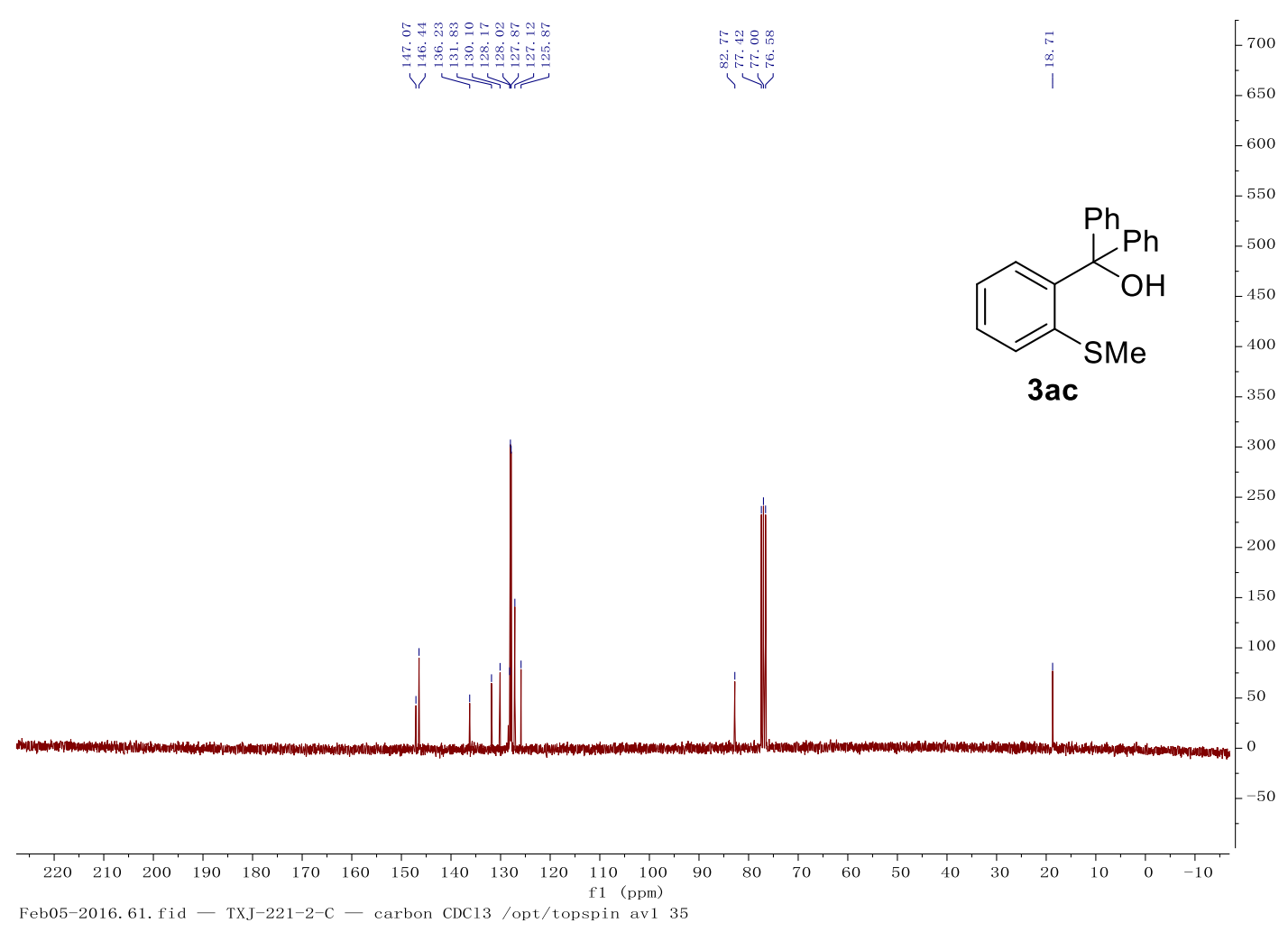




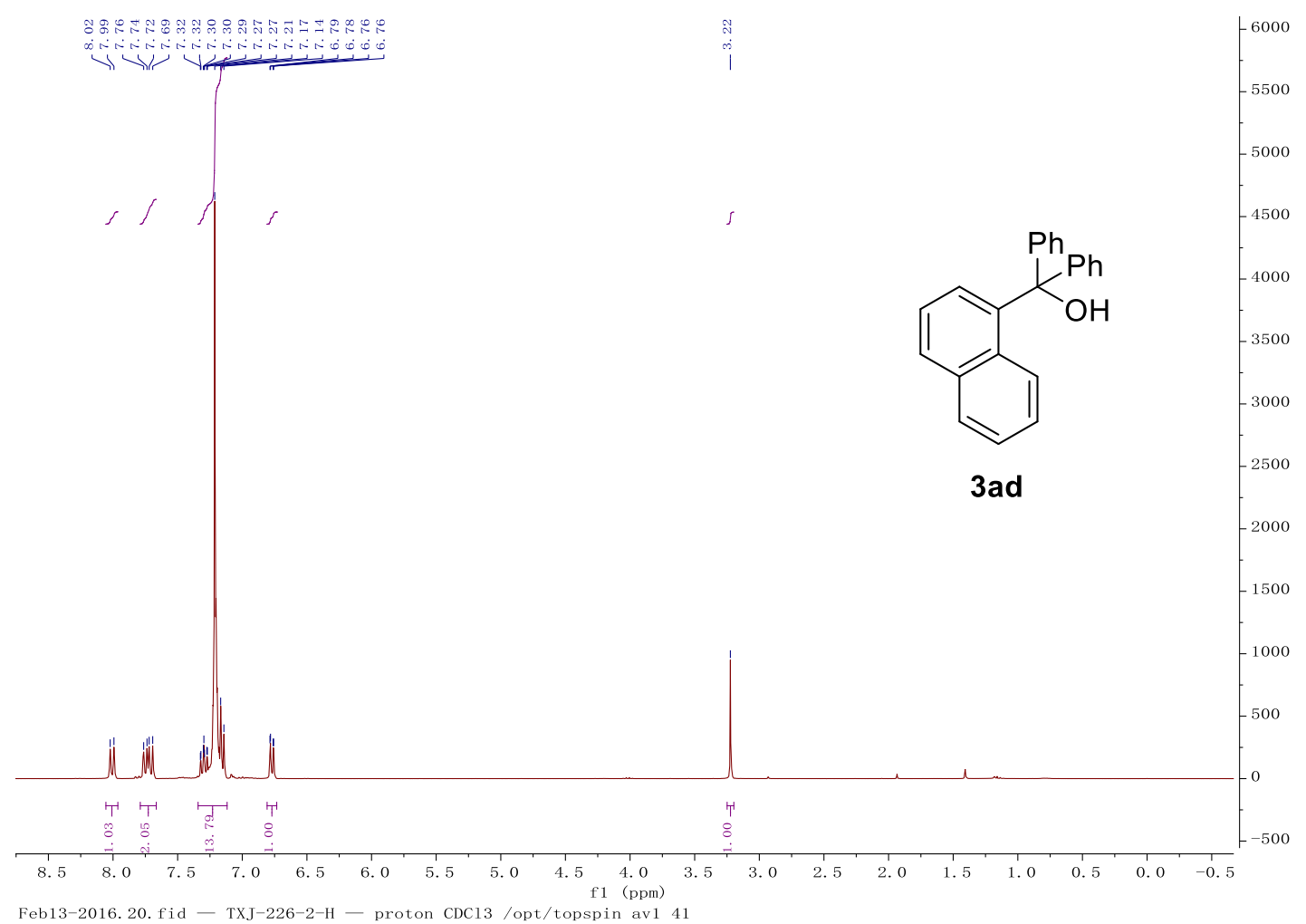

Feb13-2016. 20.fid - TXJ-226-2-H - proton CDC13/opt/topspin av1 41

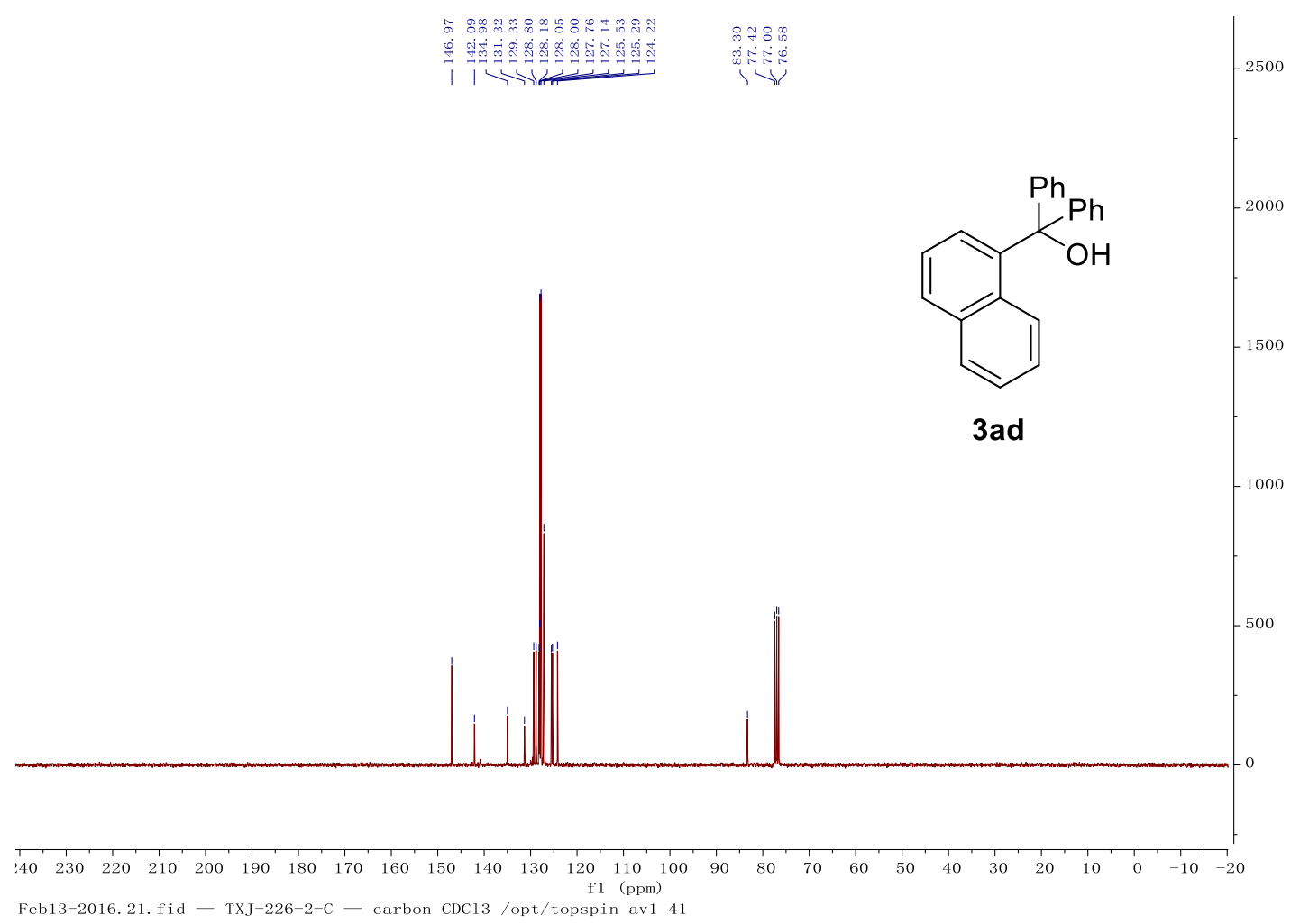




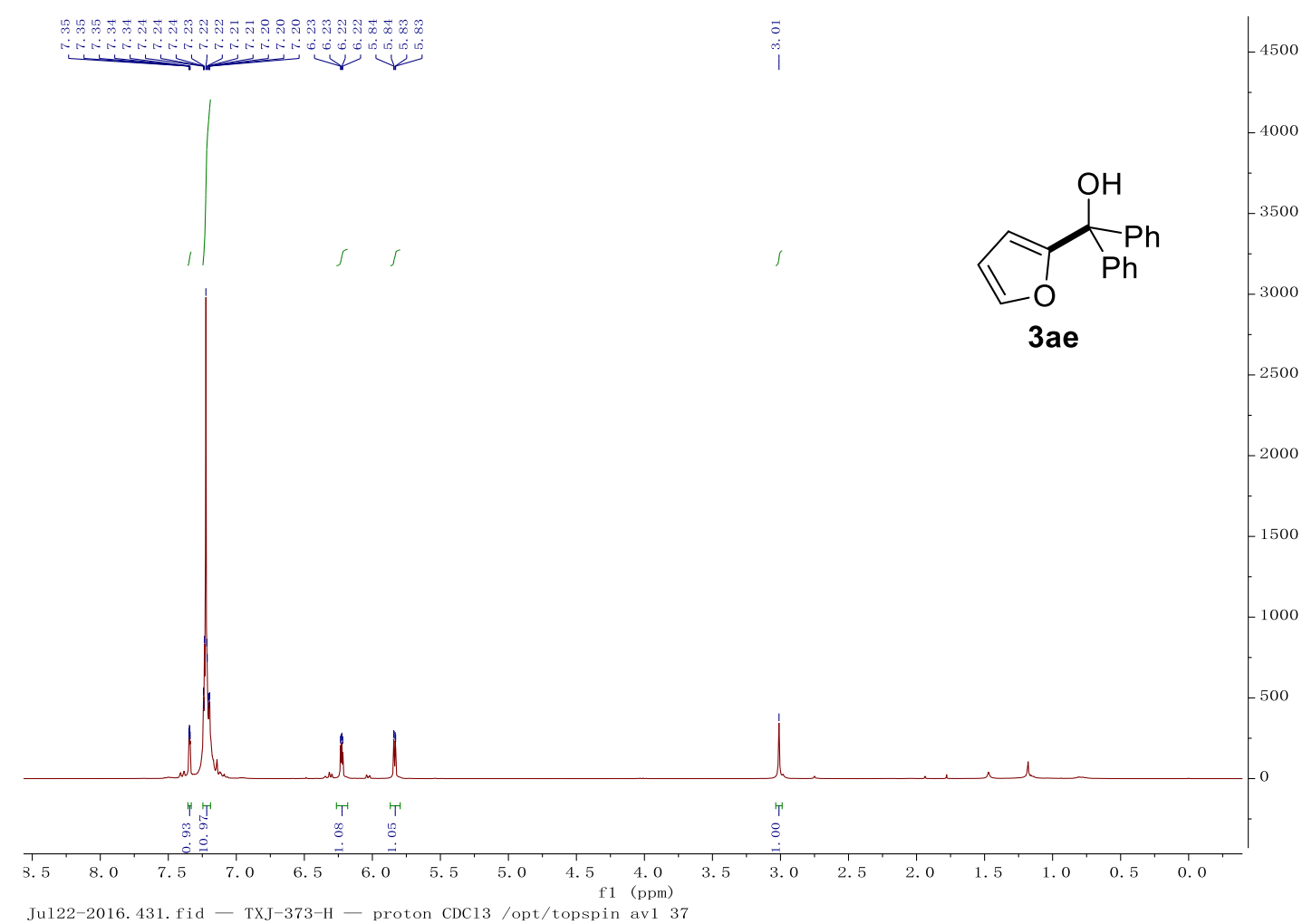

Ju122-2016. 431. fid - TXJ-373-H - proton CDC13/opt/topspin av1 37

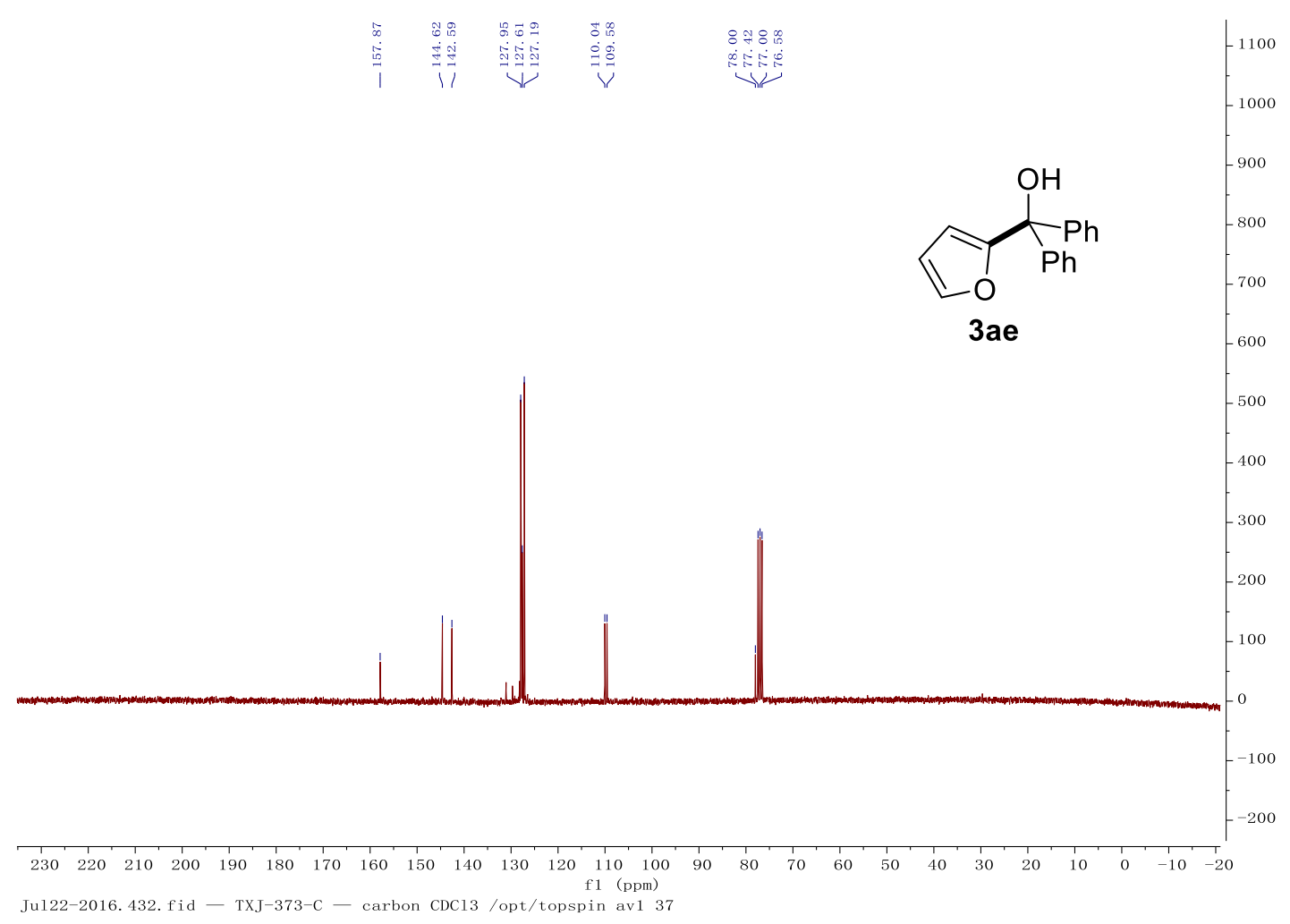




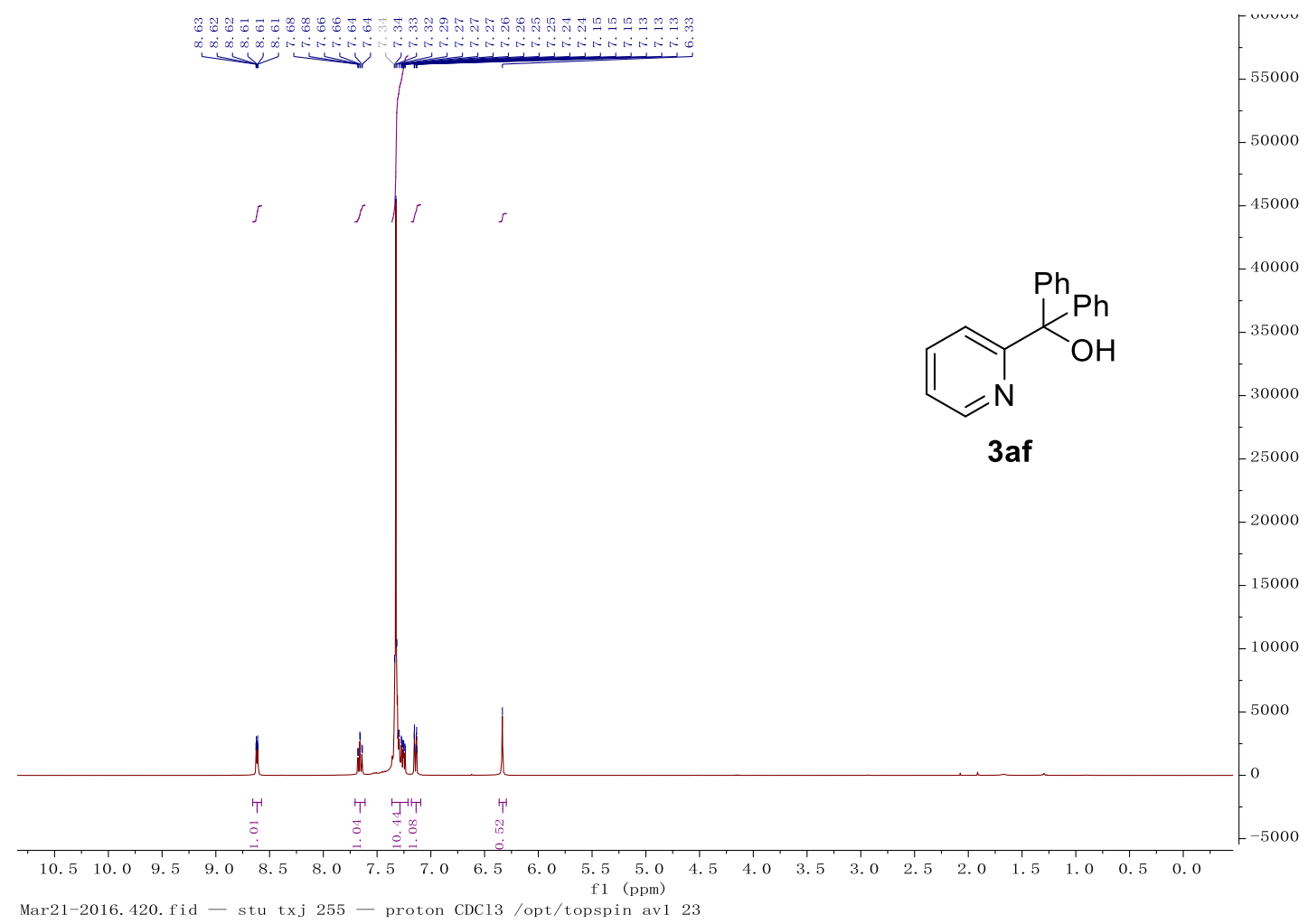

Mar21-2016. 420. fid - stu txj 255 - proton CDC13/opt/topspin av1 23

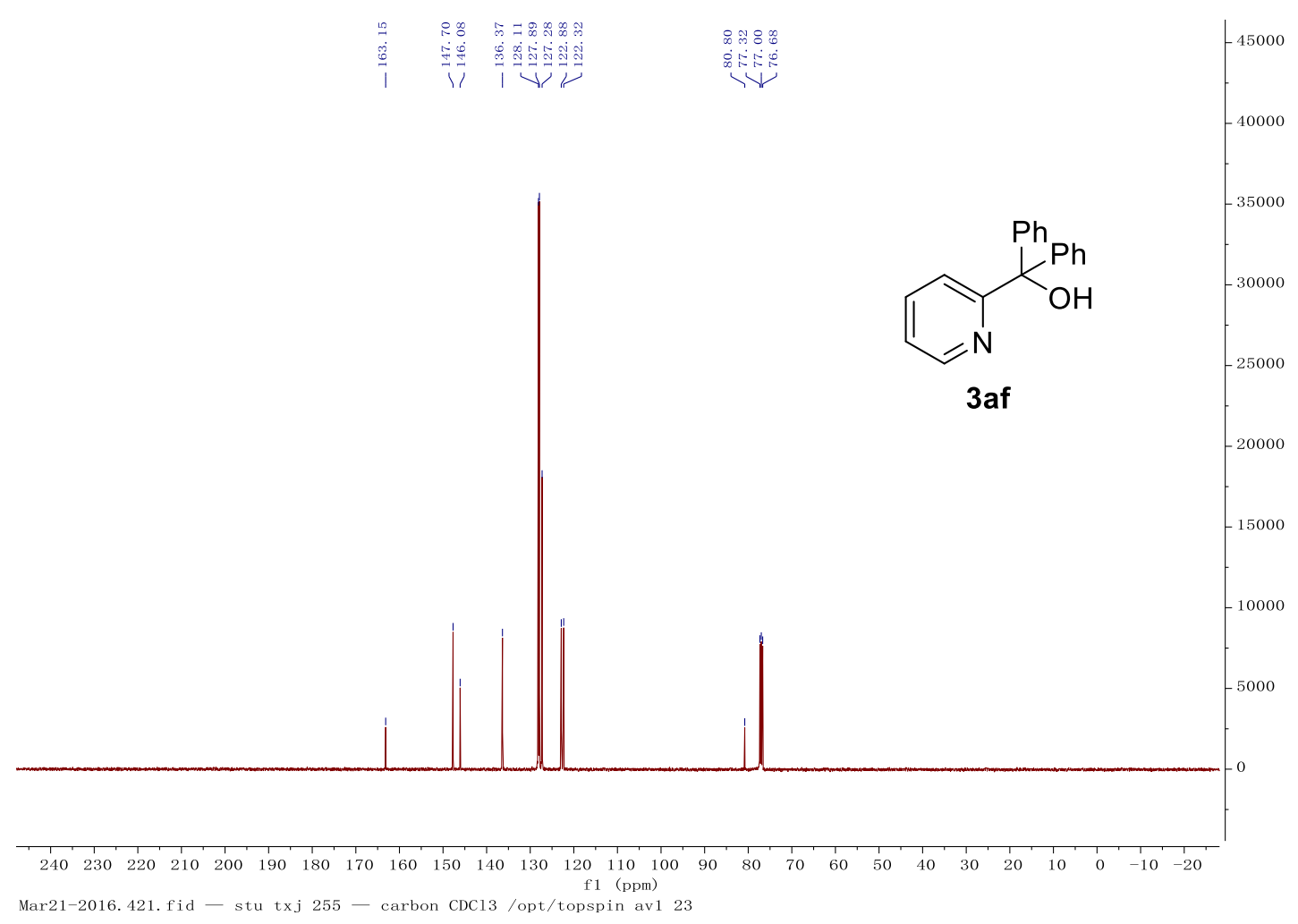



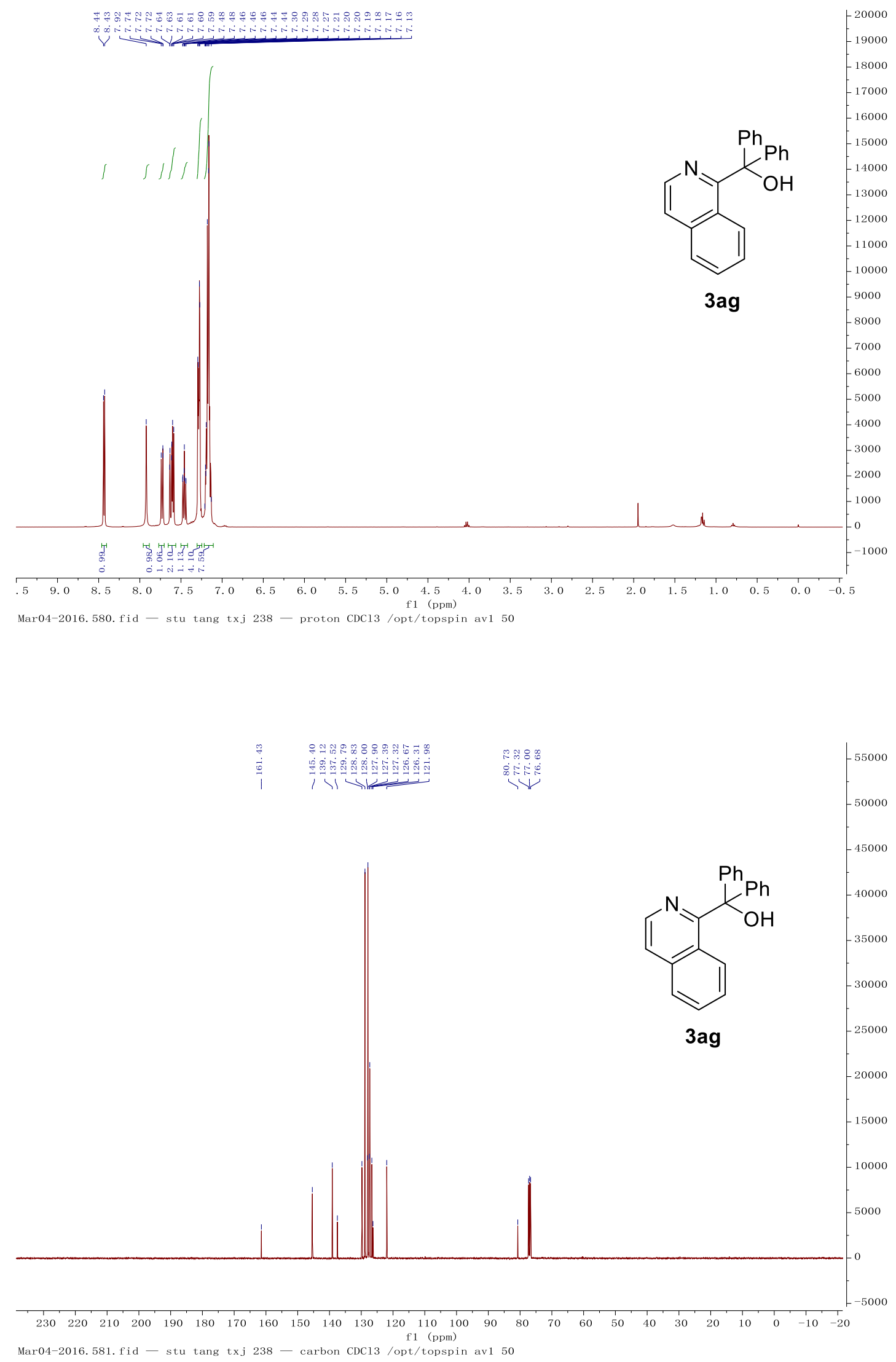


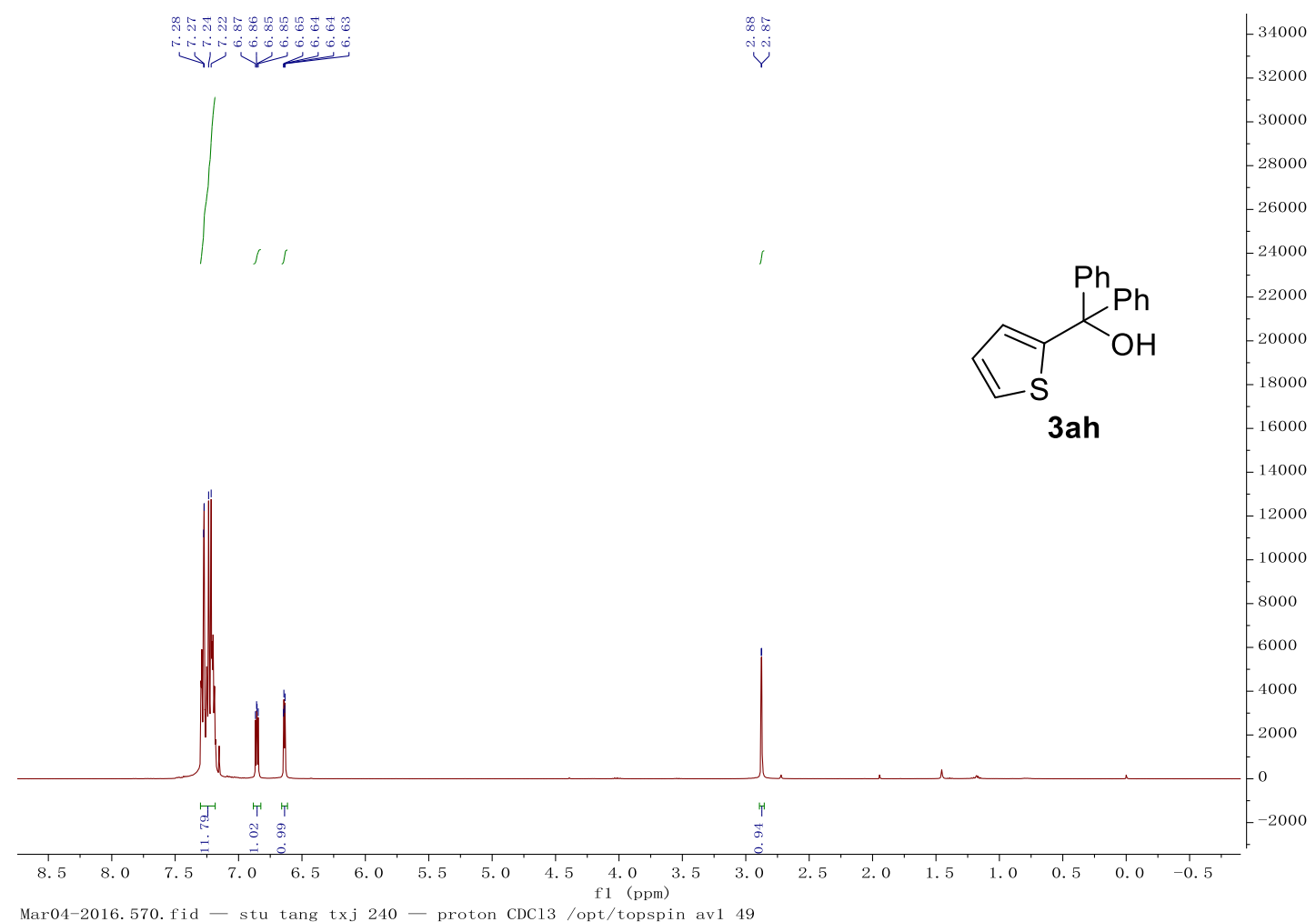

Mar04-2016.570. fid - stu tang txj 240 - proton CDC13/opt/topspin av1 49

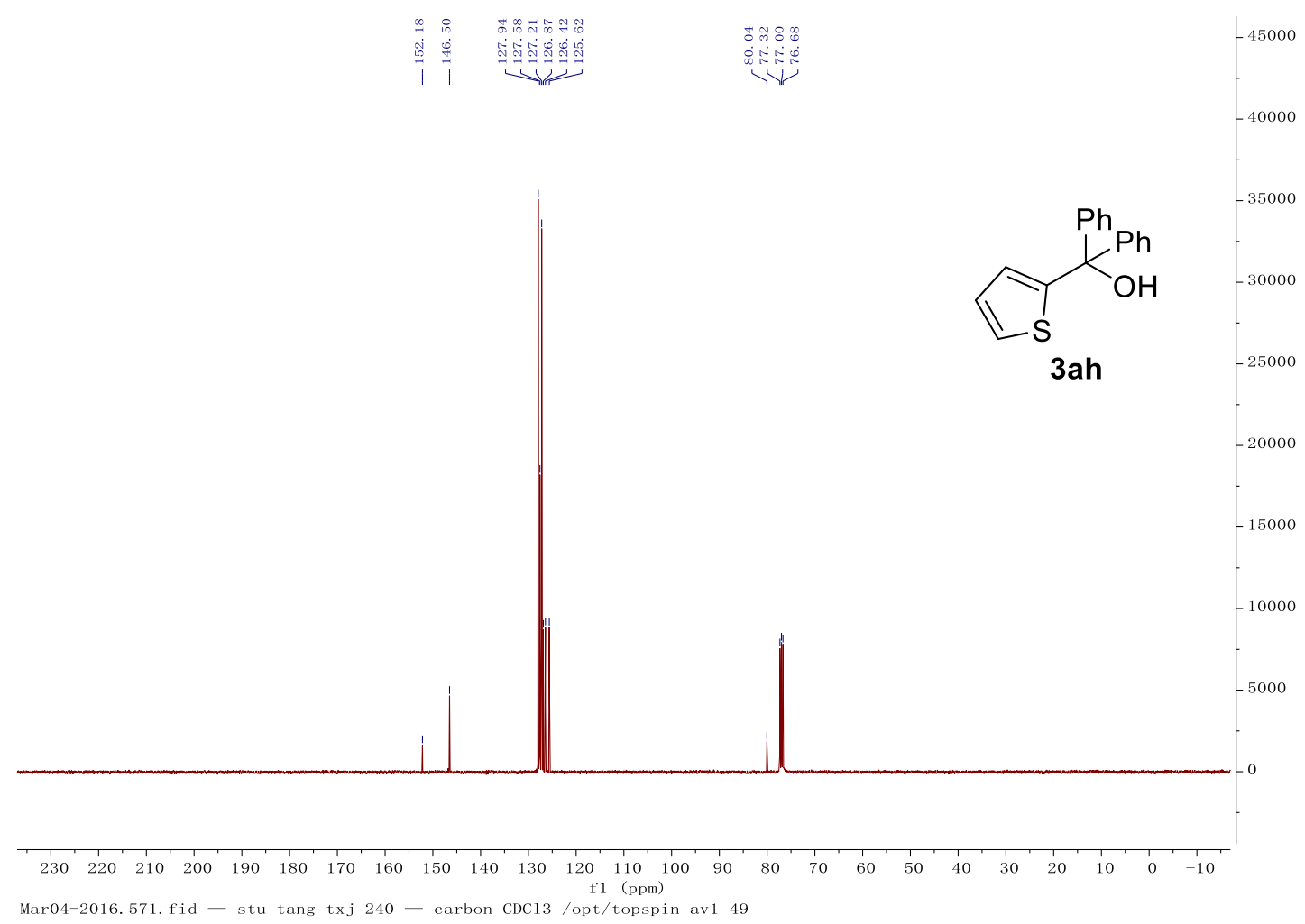




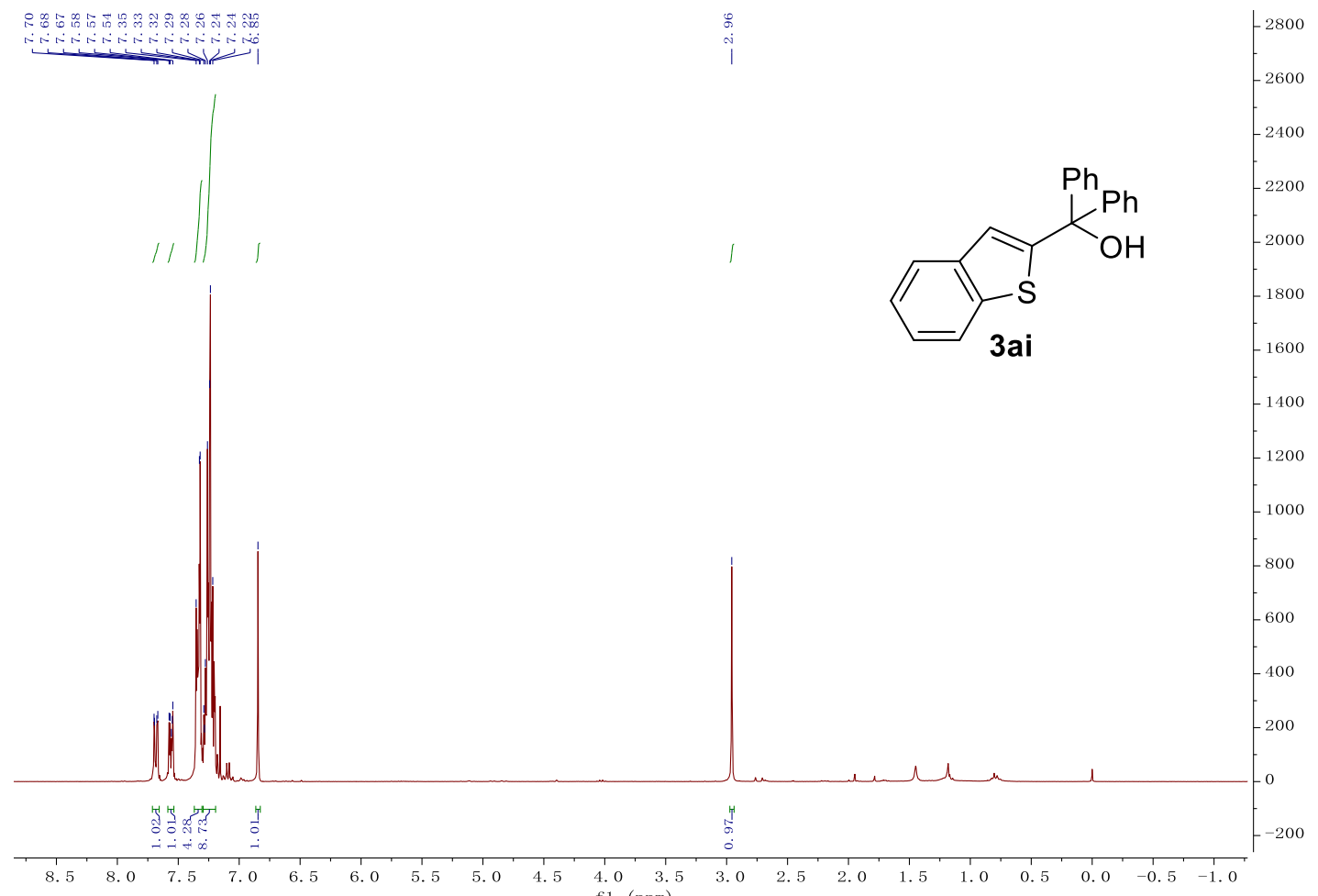

Mar31-2016. 330. fid - TXJ-262-H - proton CDC13/opt/topspin av1 40

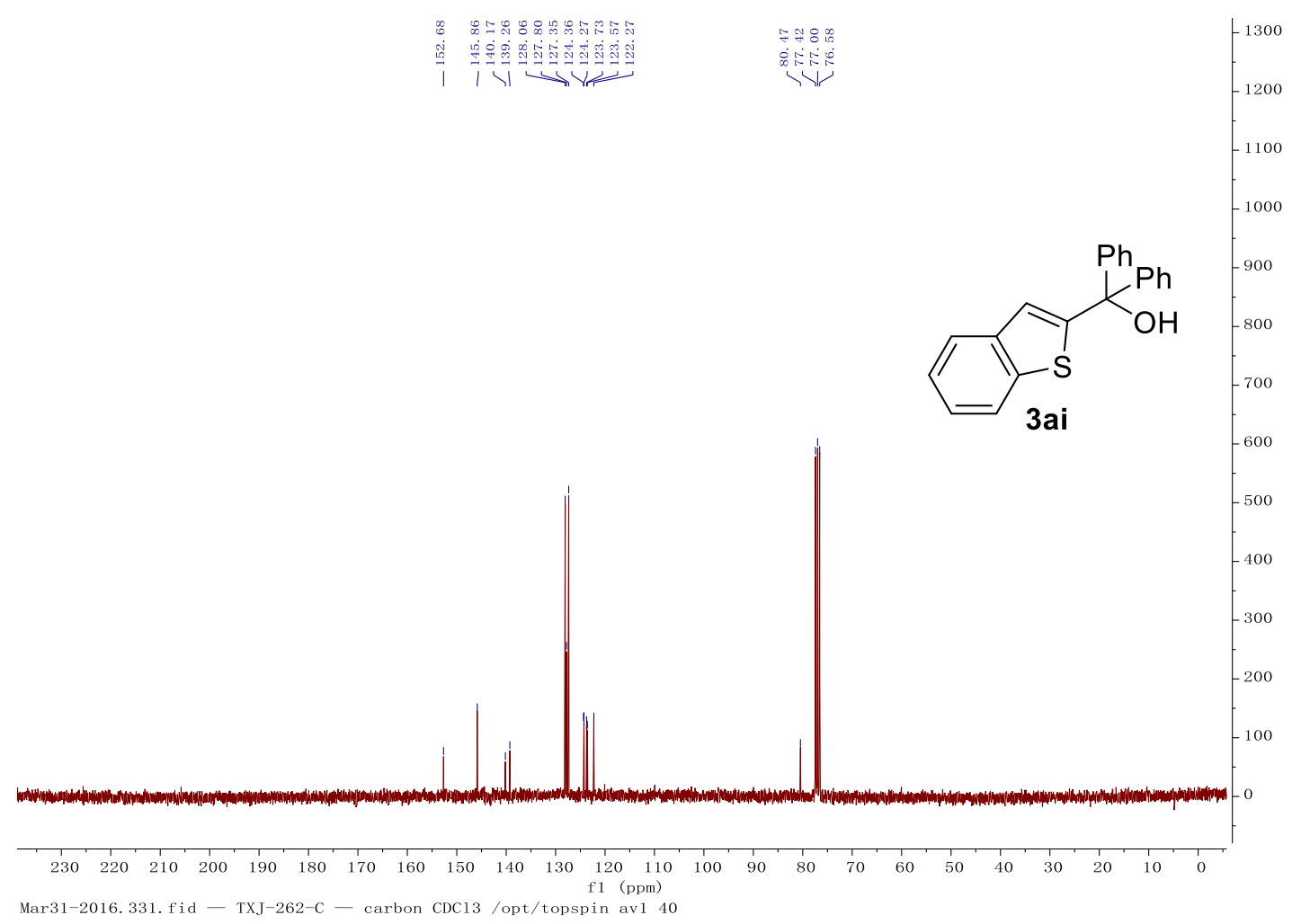




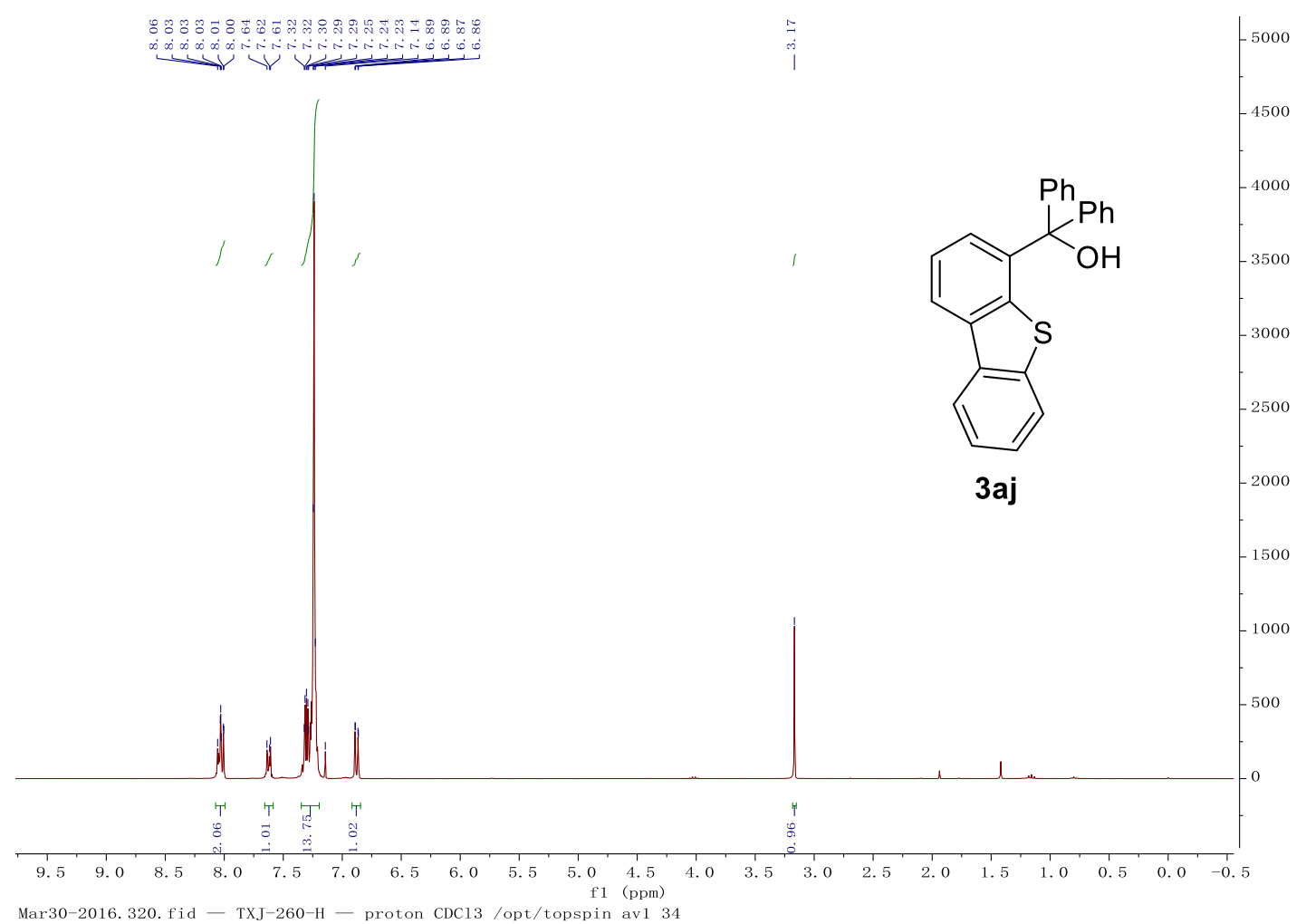

Mar30-2016. 320. fid - TXJ-260-H - proton CDC13/opt/topspin av1 34

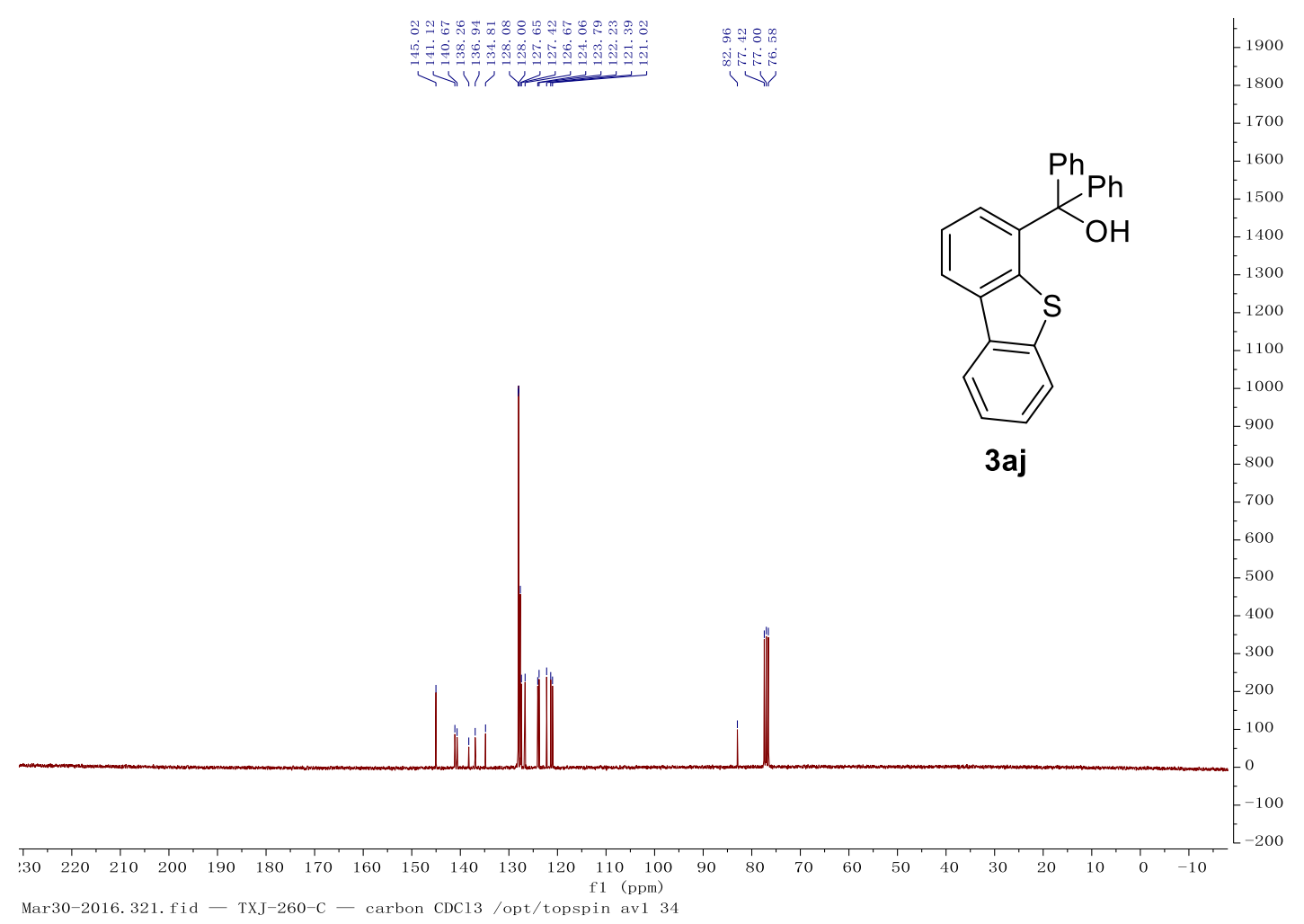




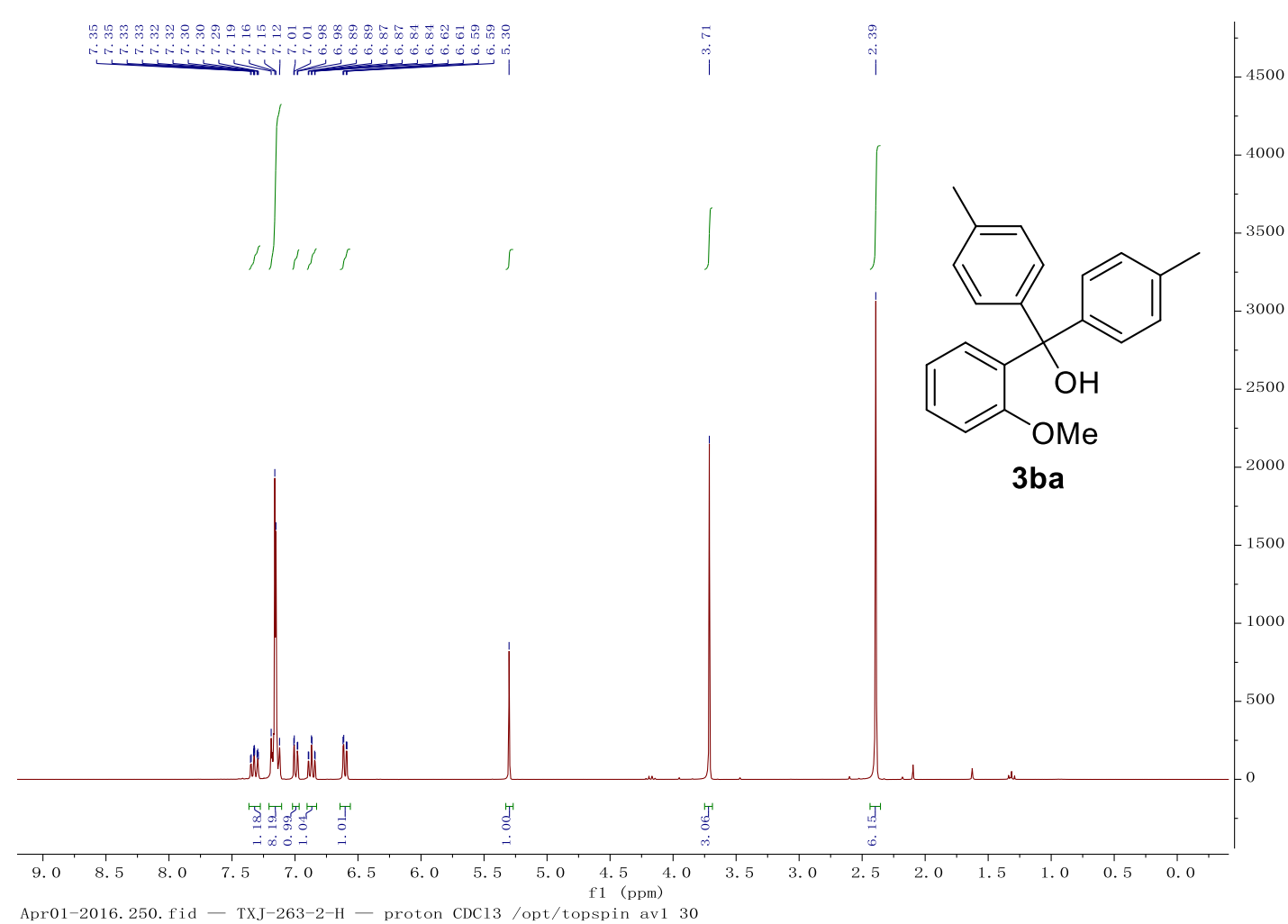

Apro1-2016. 250. fid - TXJ-263-2-H - proton CDC13/opt/topspin av1 30

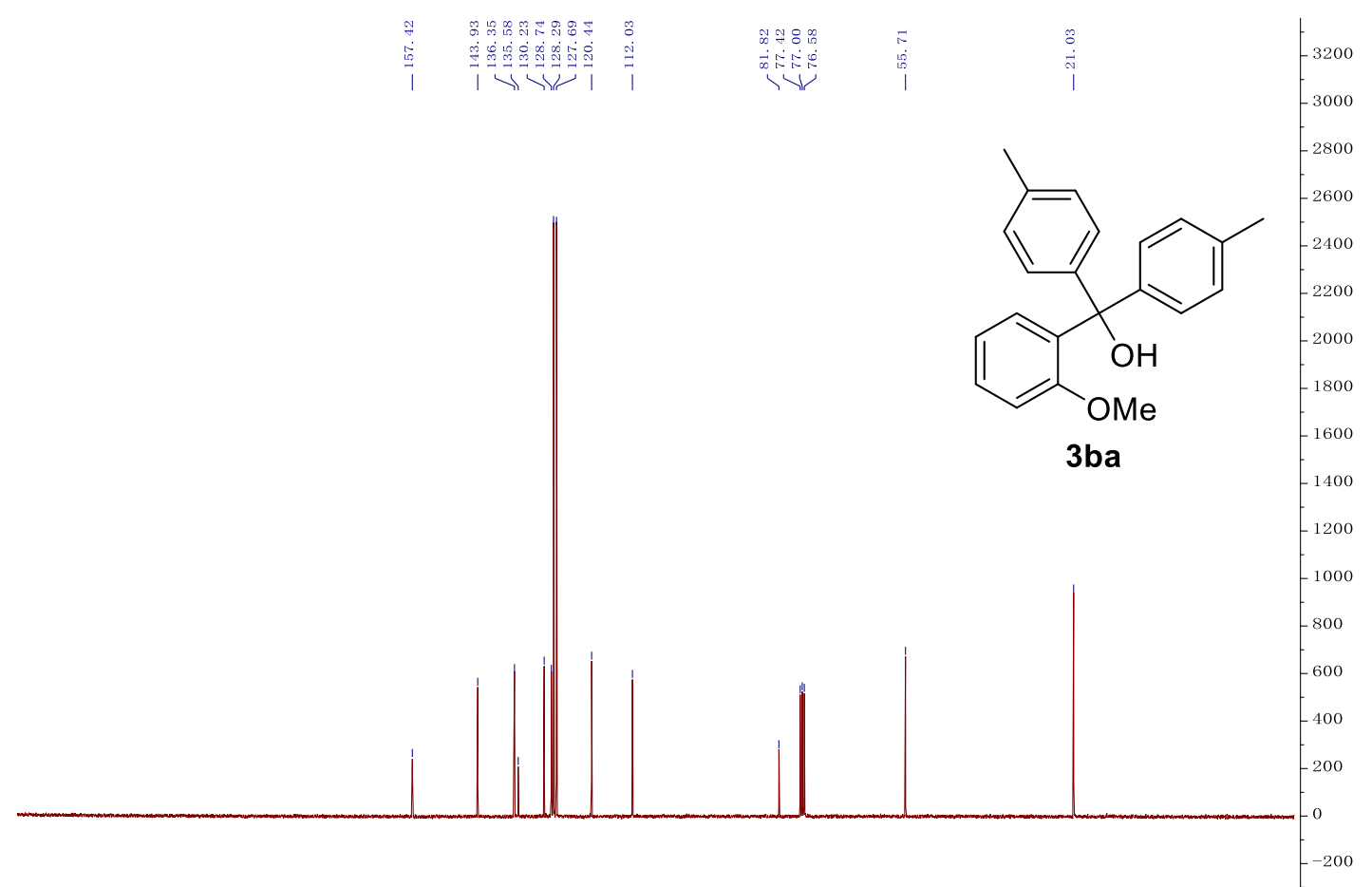

$\begin{array}{llllllllllllllllllllllllllllllllllllllllllllll}1 & 230 & 220 & 210 & 200 & 190 & 180 & 170 & 160 & 150 & 140 & 130 & 120 & 110 & 100 & 90 & 80 & 70 & 60 & 50 & 40 & 30 & 20 & 10 & 0 & -10 & -20\end{array}$ Apro1-2016. 251. fid - TXJ-263-2-C - carbon CDC13/opt/topspin av1 30 


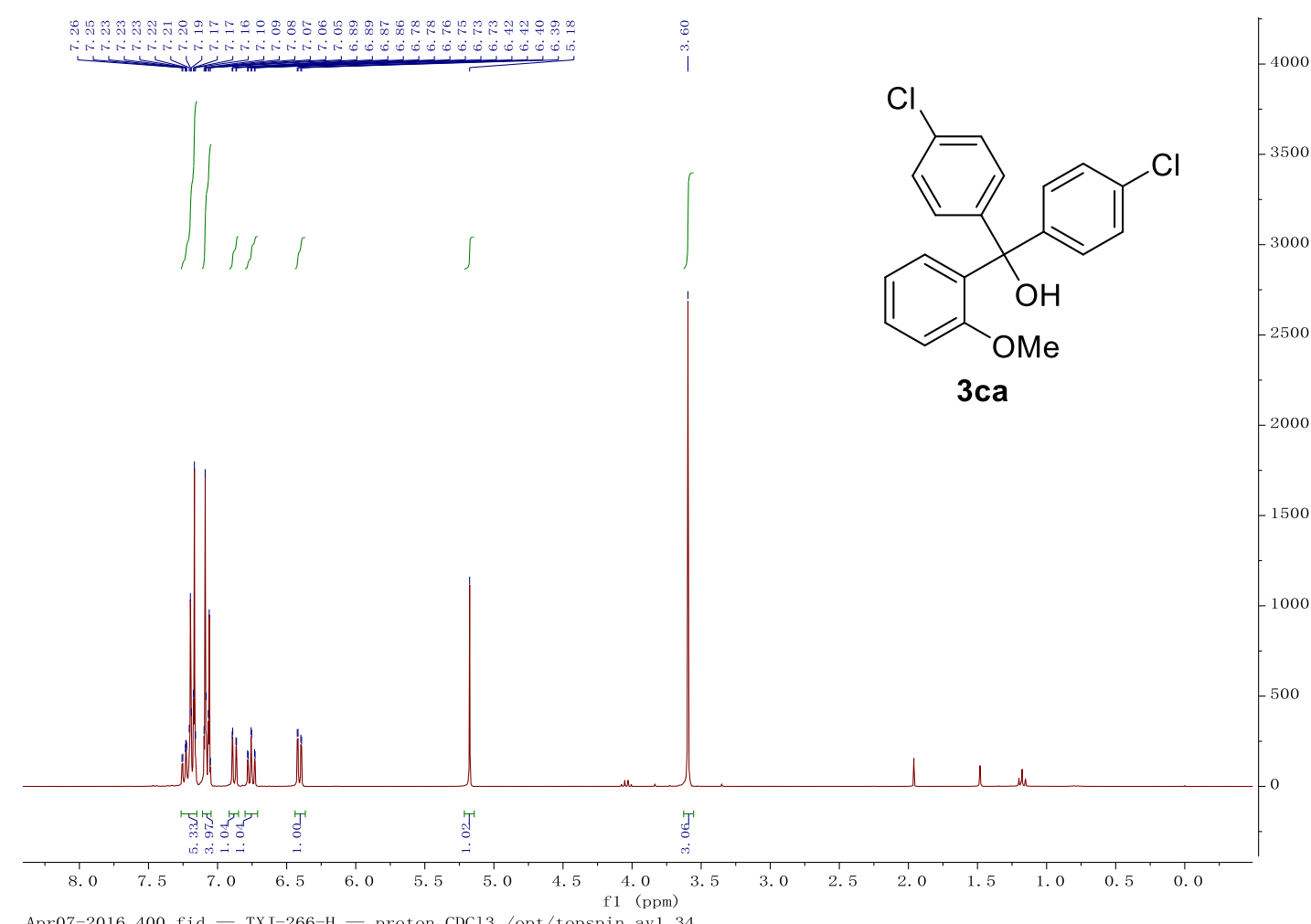

Apro7-2016. 400. fid - TXJ-266-H - proton CDC13 /opt/topspin av1 34

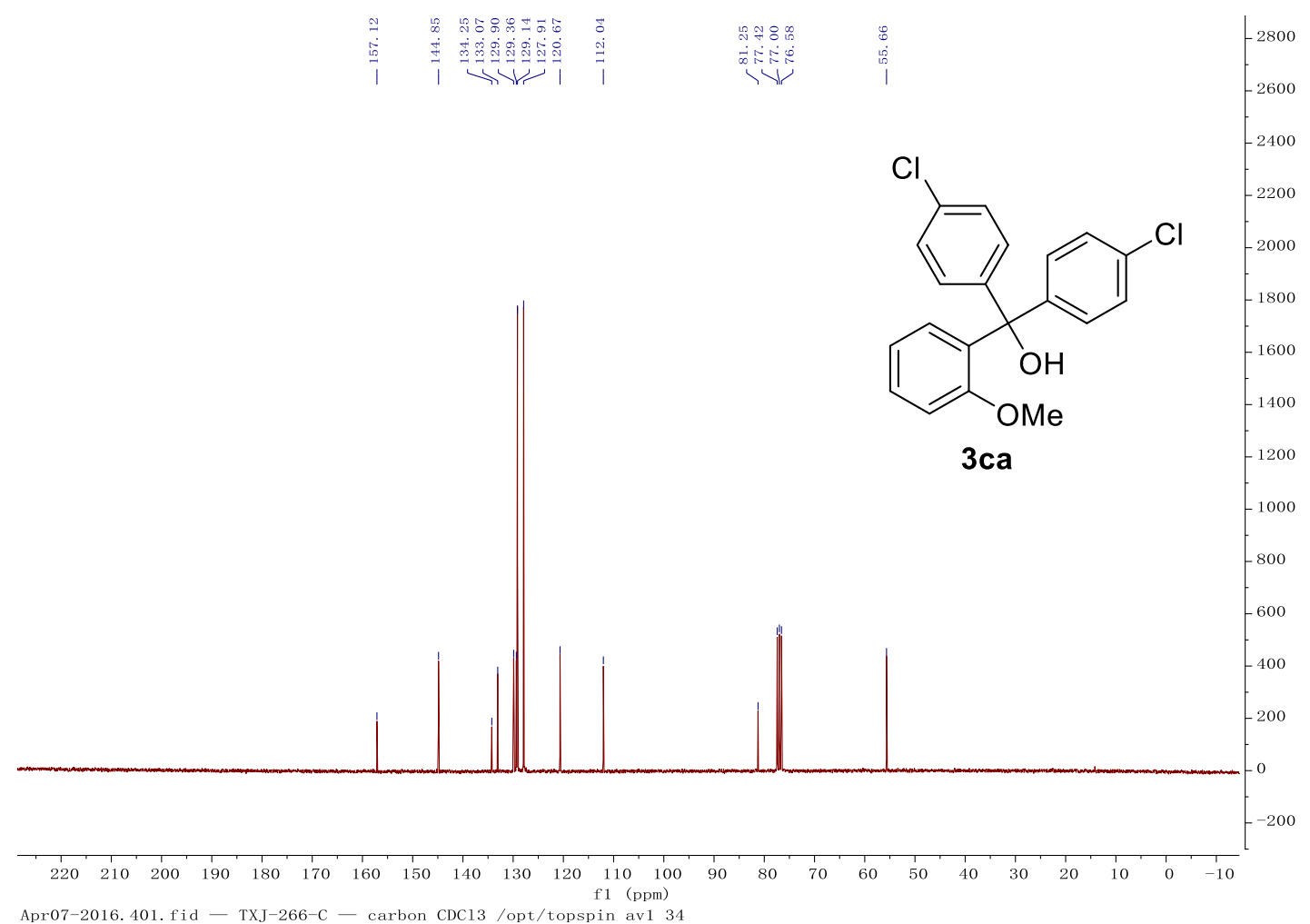

Apr07-2016. 401. fid - TXJ-266-C - carbon CDC13/opt/topspin av1 34 

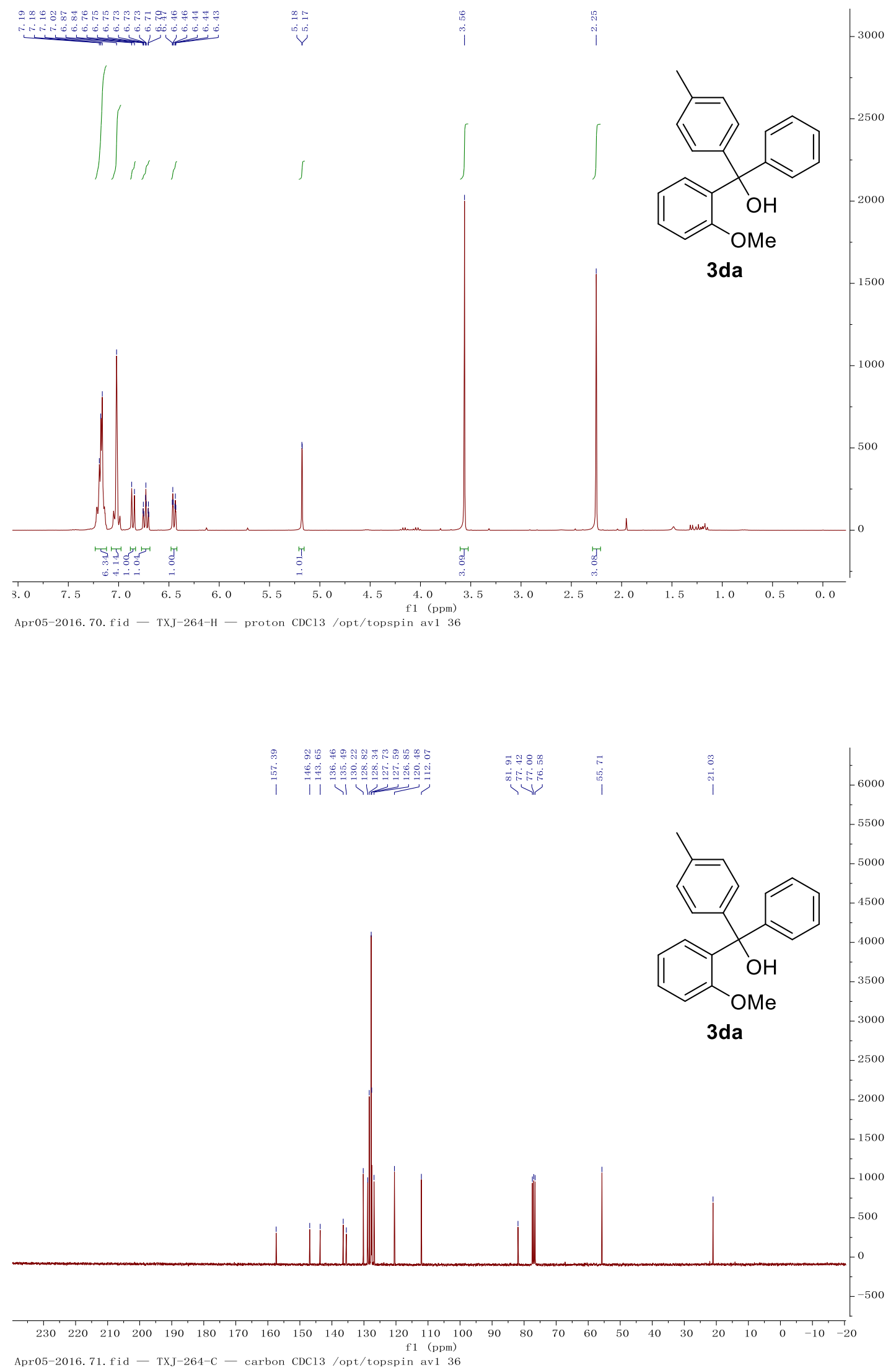


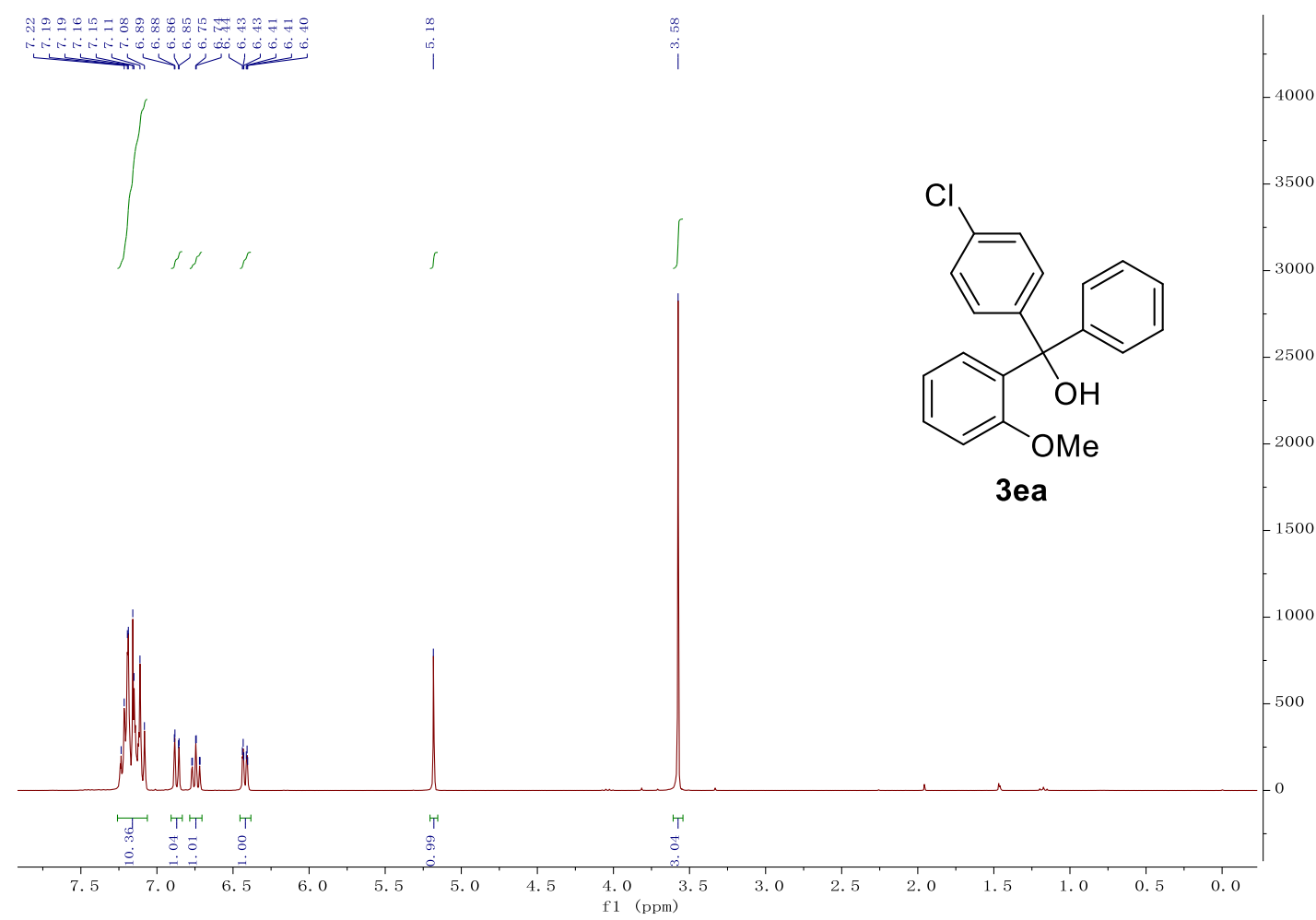

Apr14-2016. 100. fid - TXJ-269-H - proton CDC13 /opt/topspin av1 30

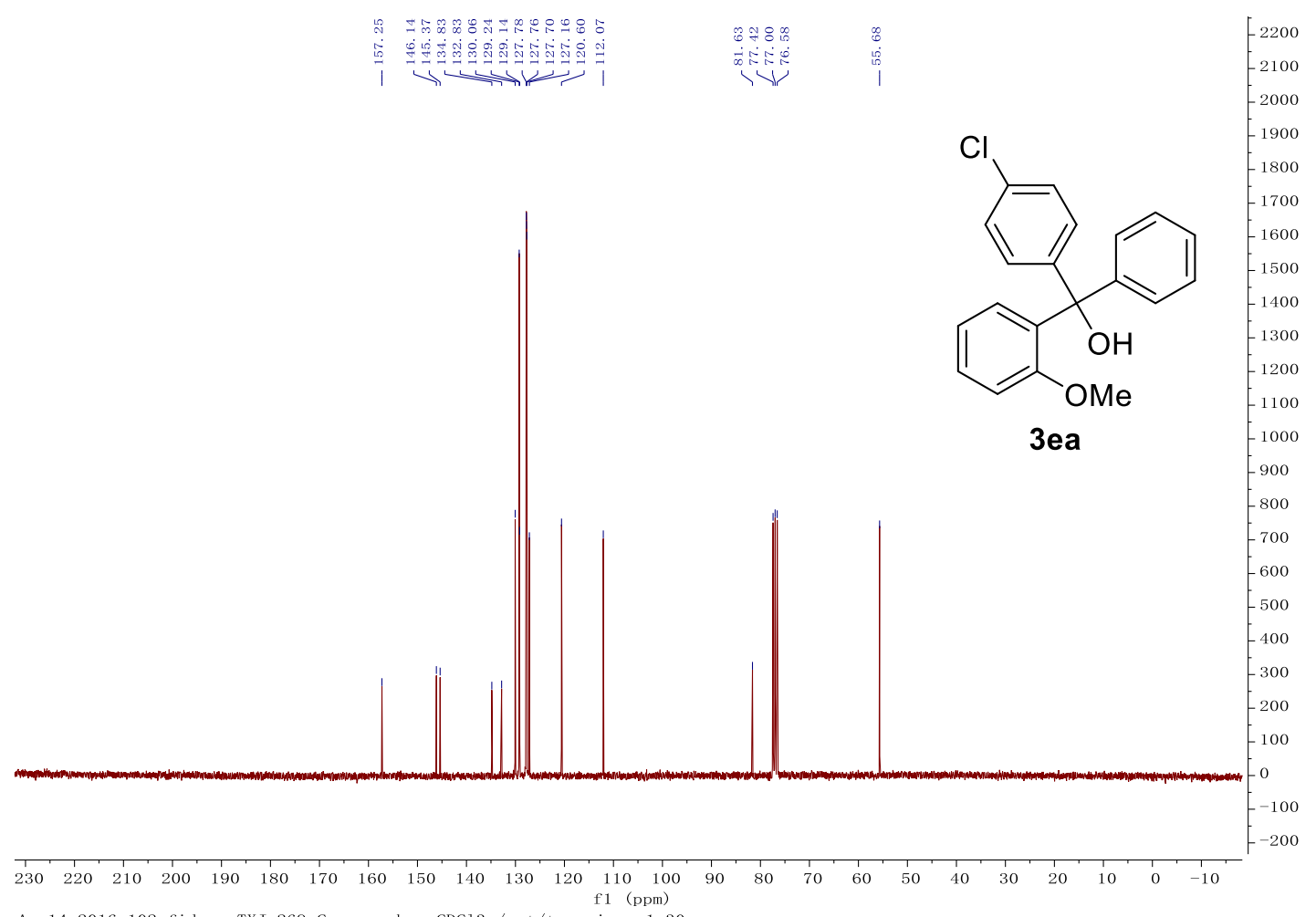
Apr14-2016. 102. fid - TXJ-269-C - carbon CDC13 /opt/topspin av1 30 


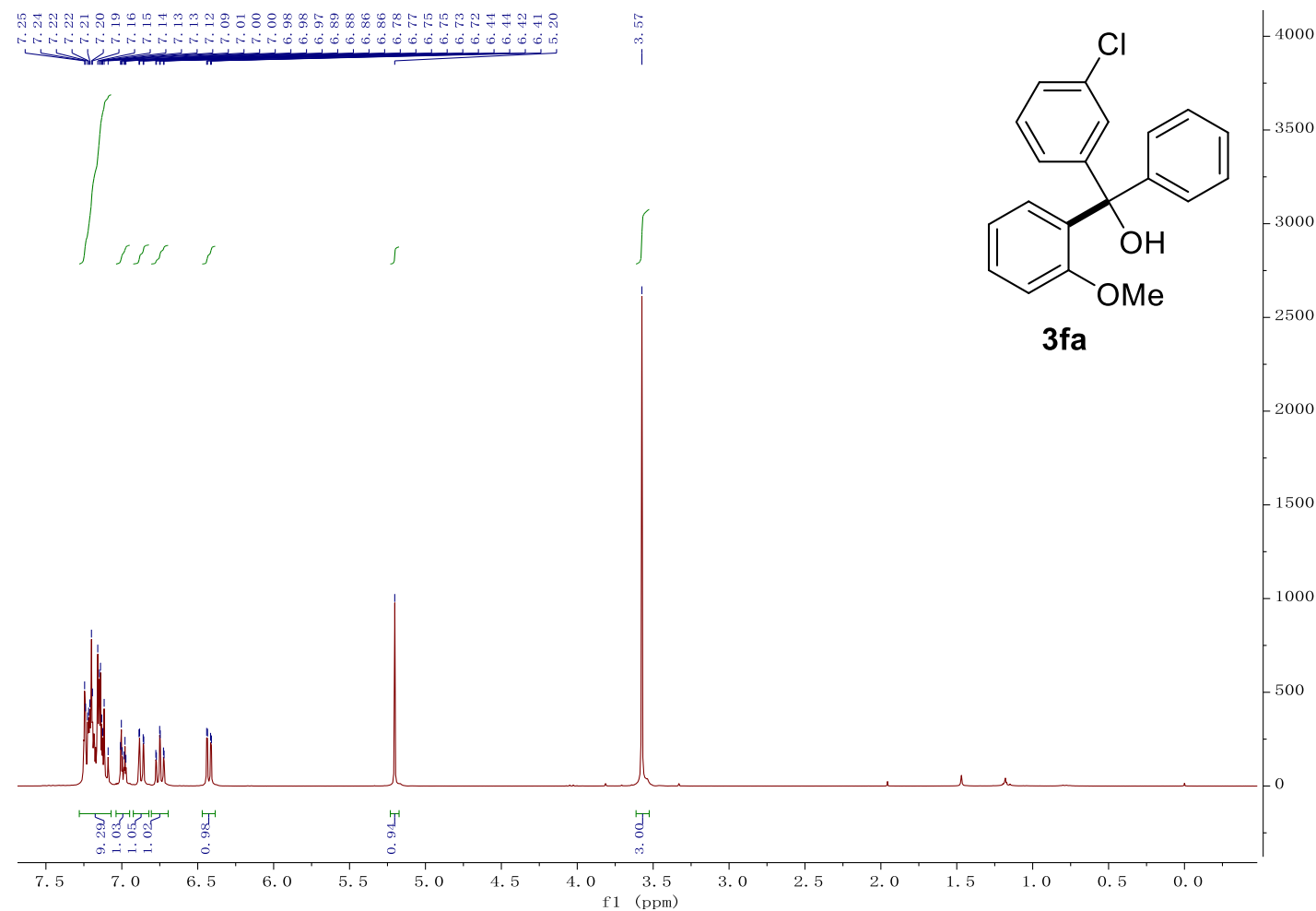

Ju121-2016. 250. fid - TXJ-372-H - proton CDC13 /opt/topspin av1 34

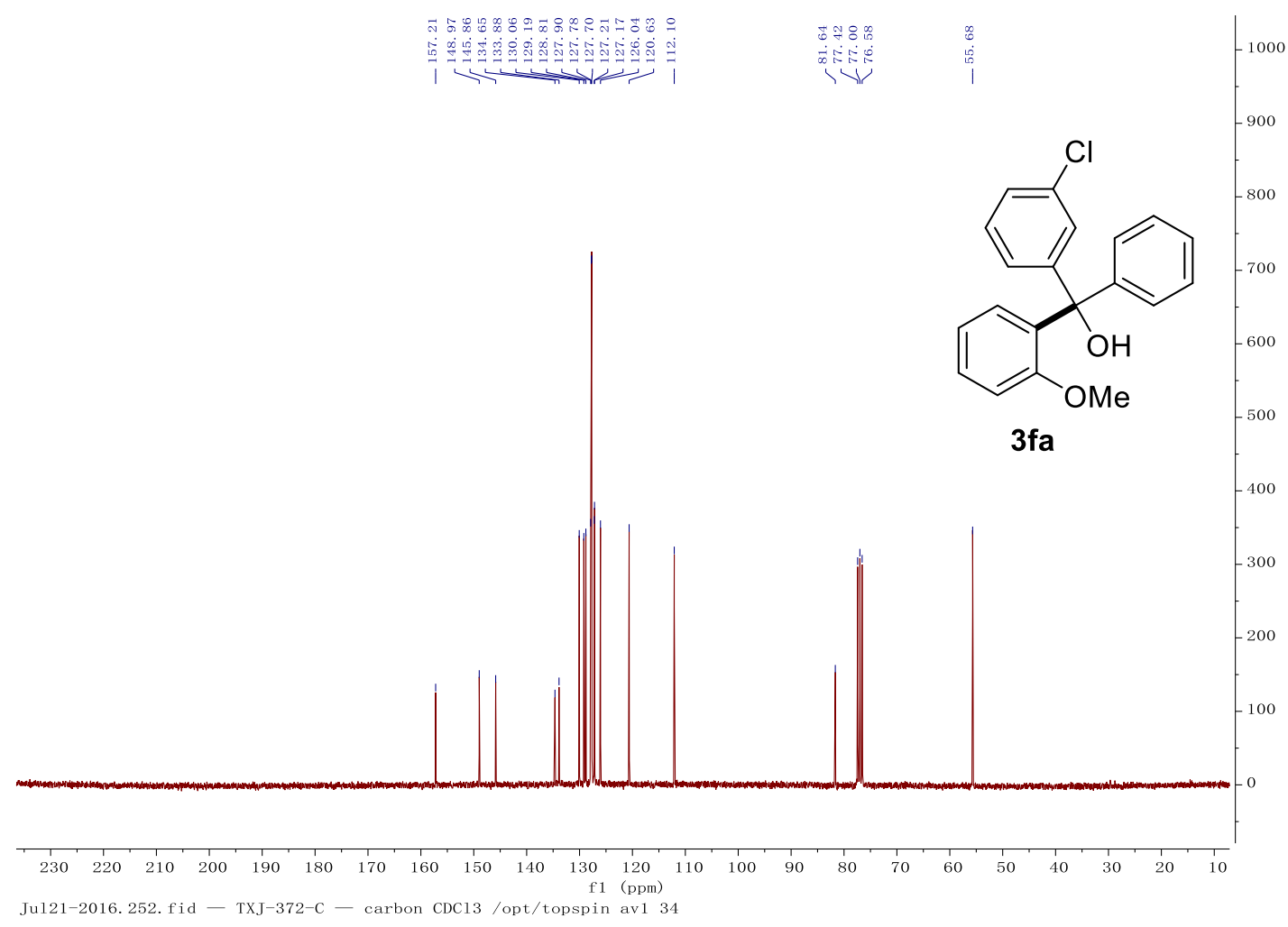




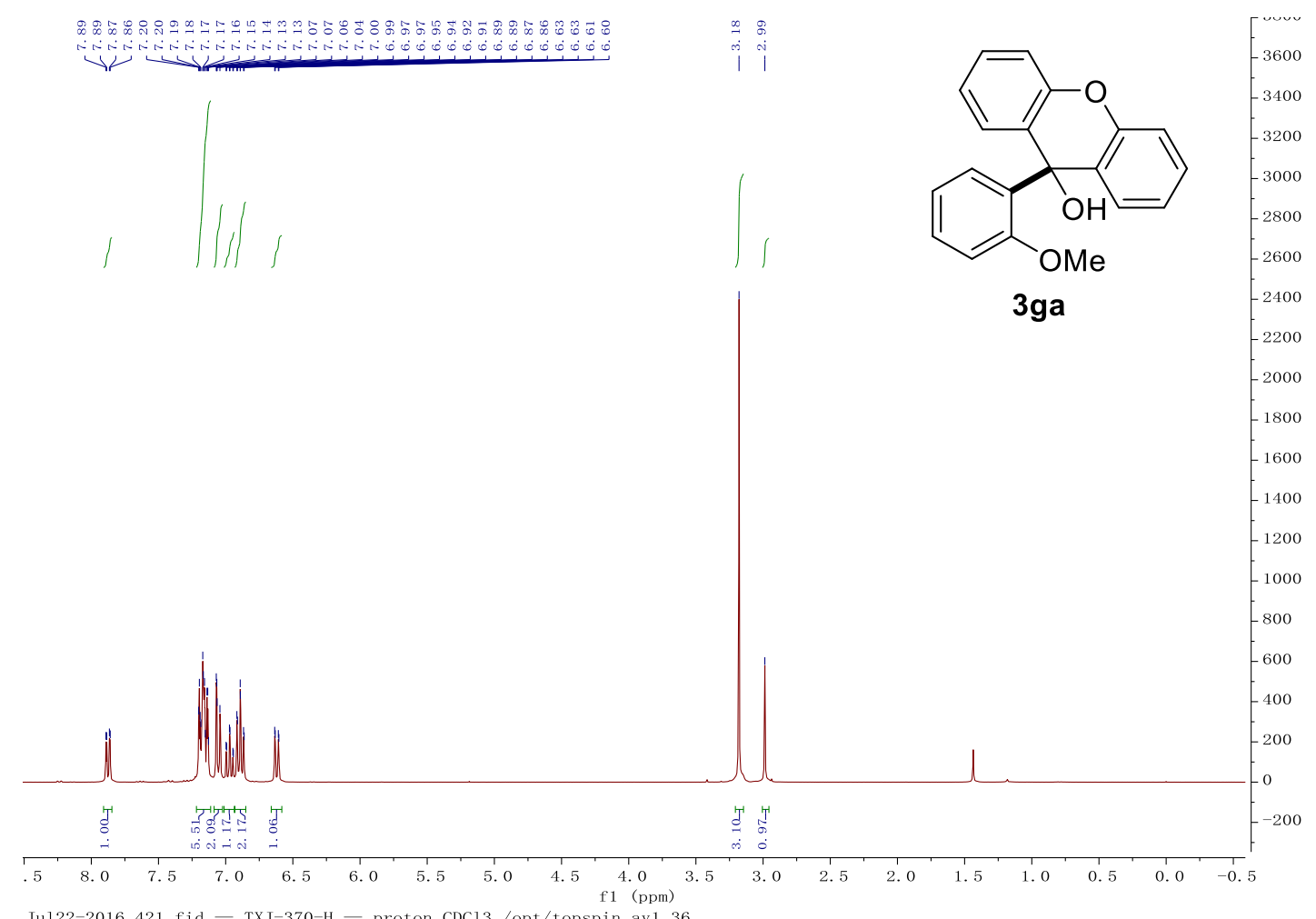

Ju122-2016. 421. fid - TXJ-370-H - proton CDC13/opt/topspin av1 36

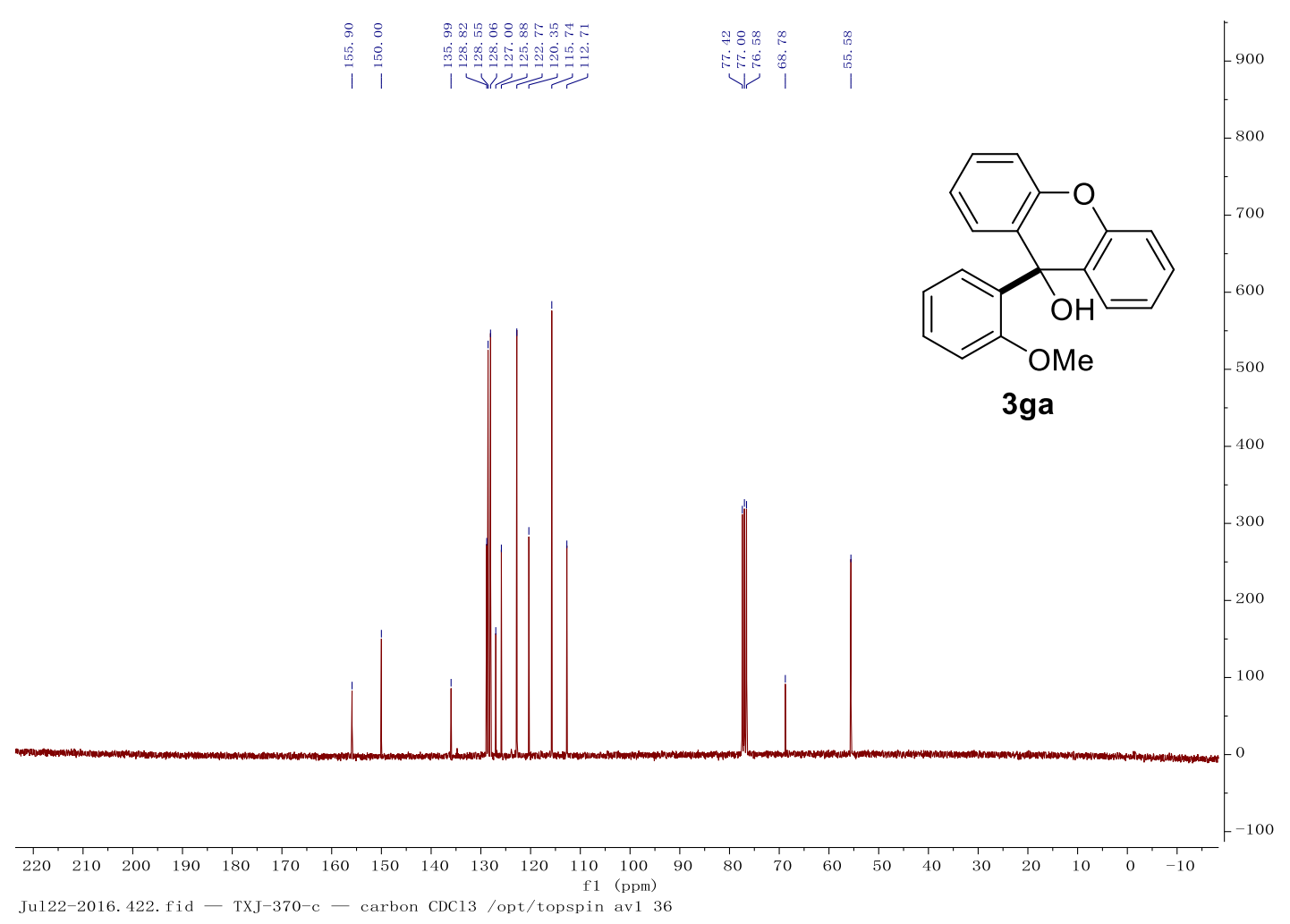



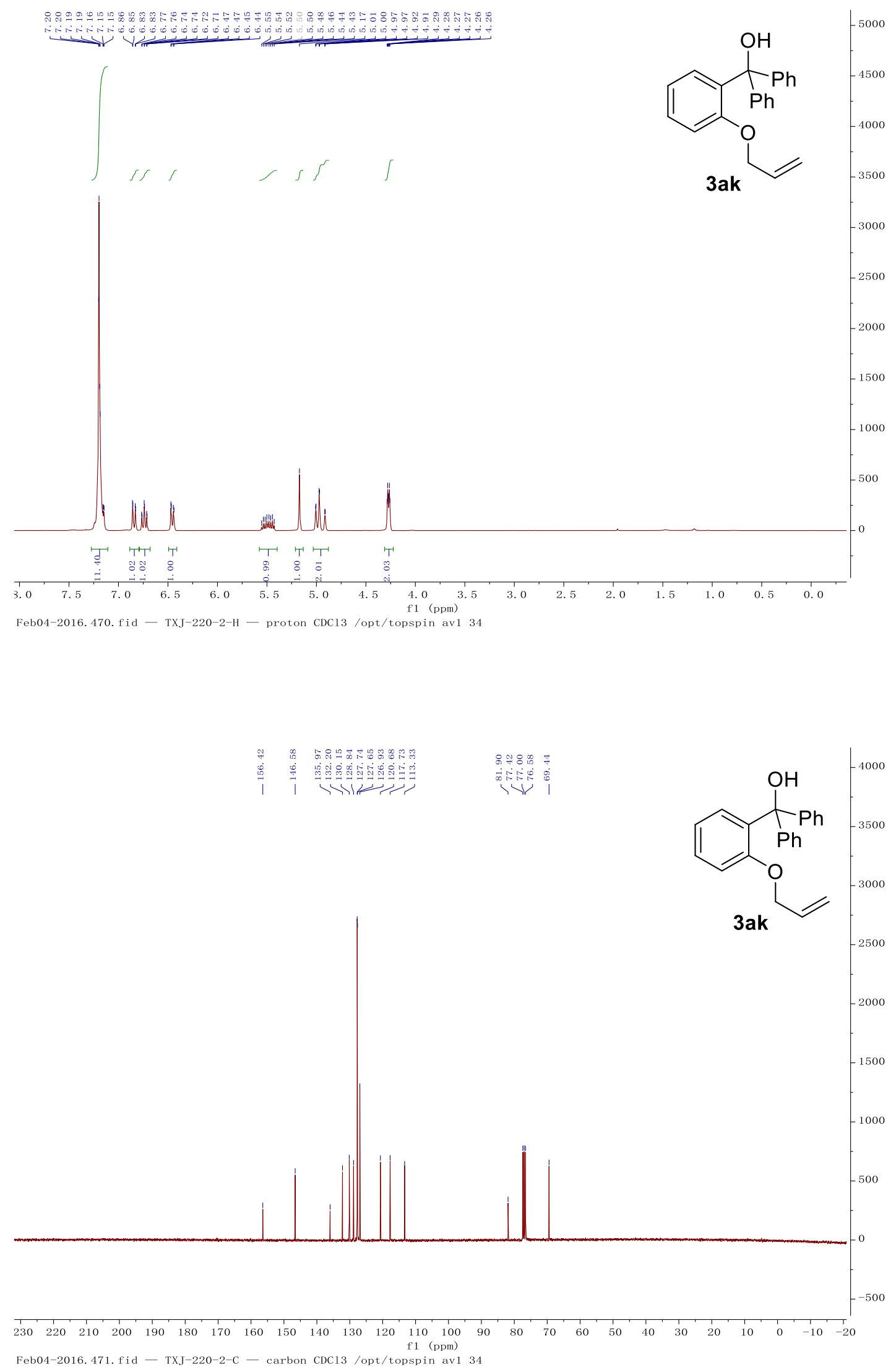


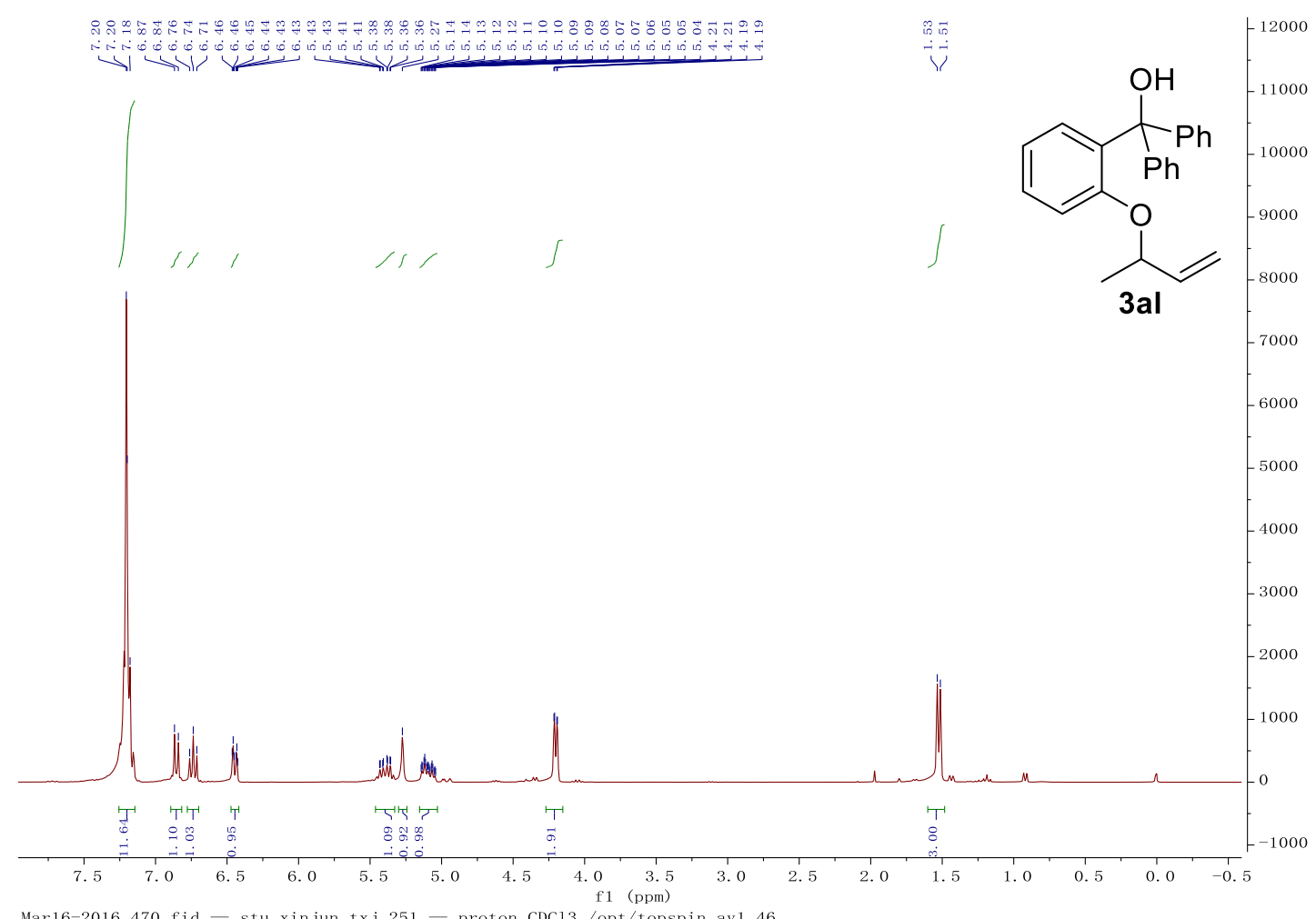

Mar16-2016. 470. fid - stu xinjun txj 251 - proton CDC13 /opt/topspin av1 46

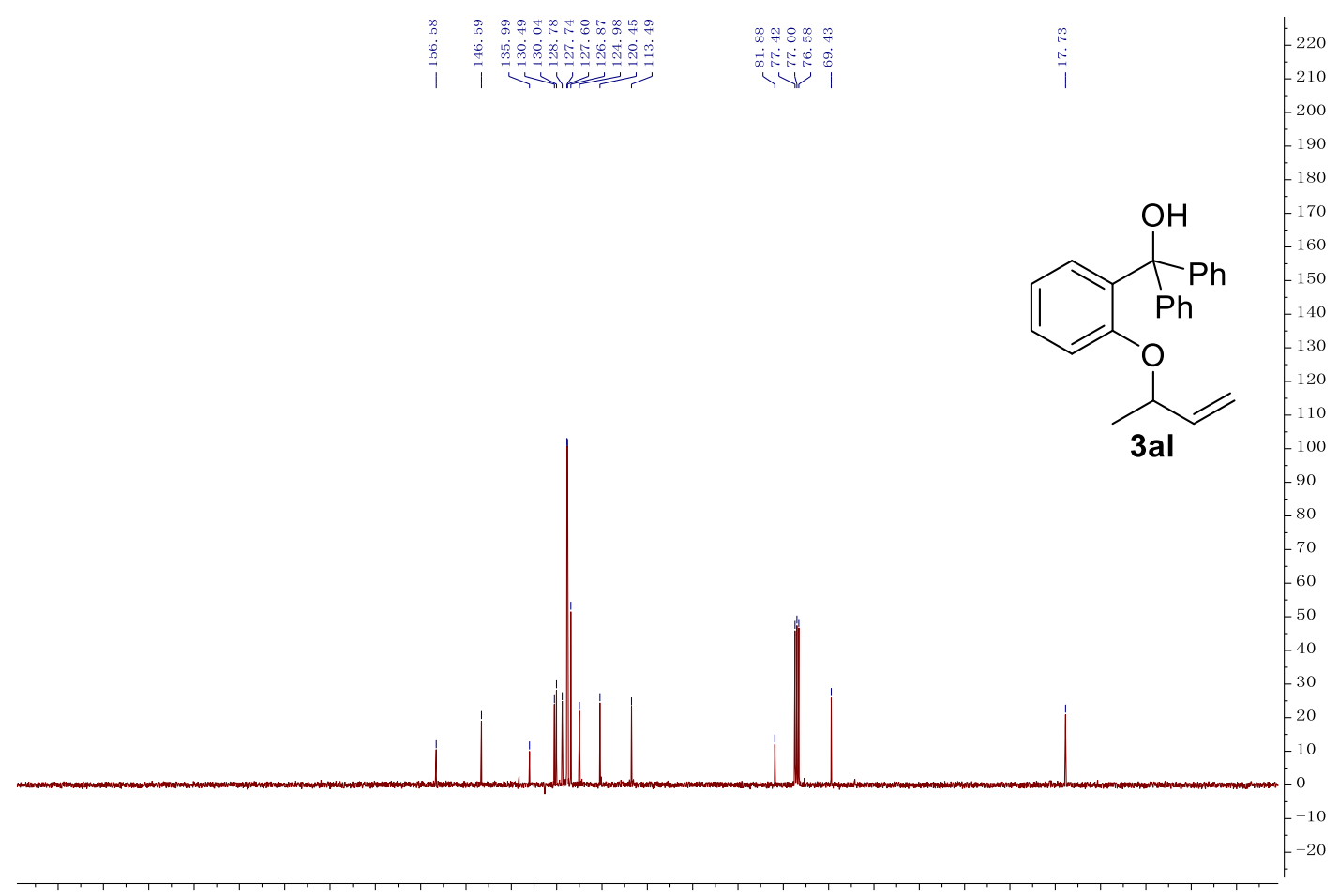

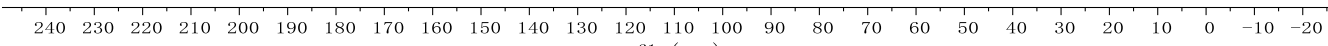
Mar16-2016. 471. fid - stu xinjun txj 251 - carbon CDC13 /opt/topspin av1 46 


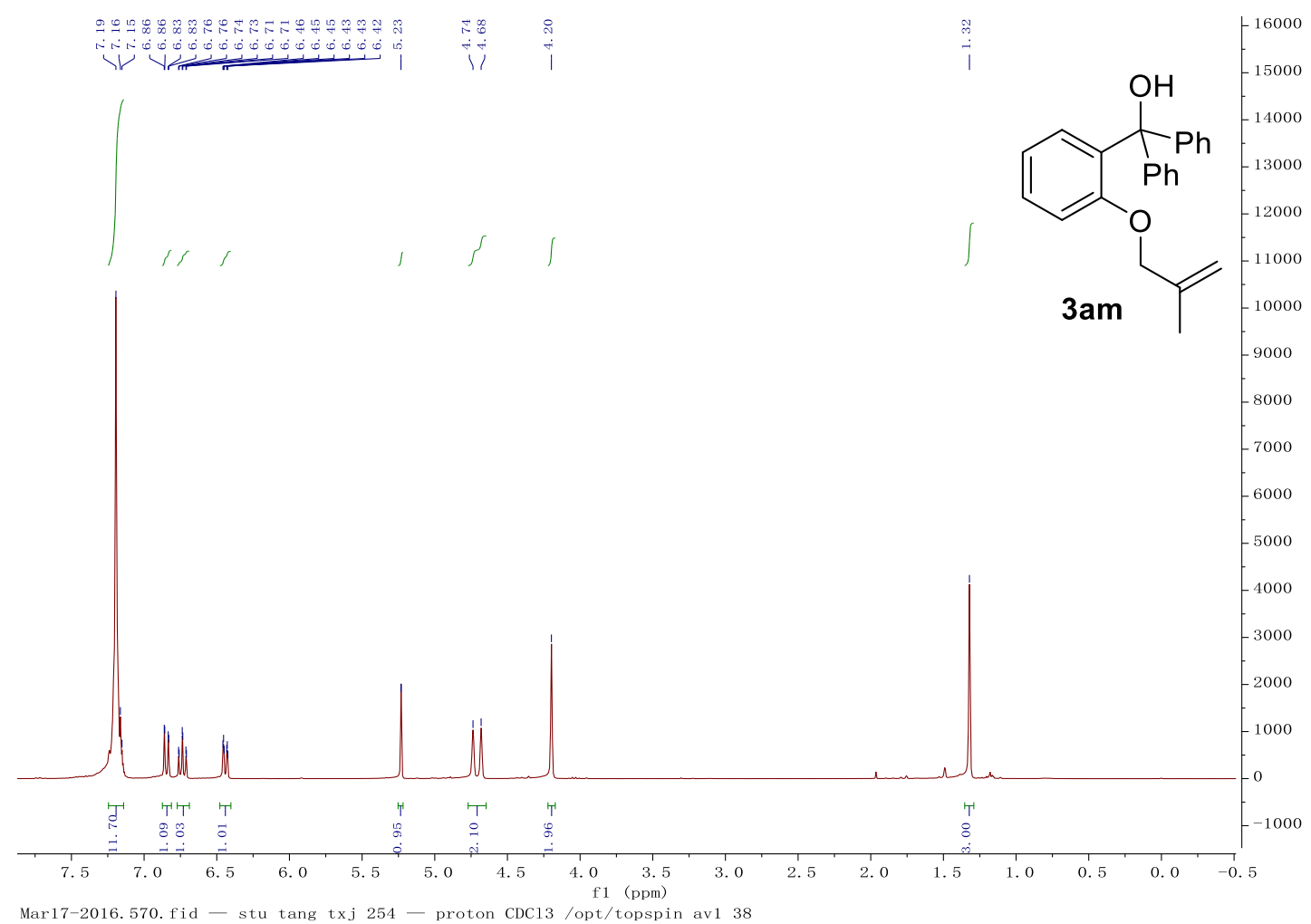

Mar17-2016.570.fid - stu tang txj 254 - proton CDC13 /opt/topspin av1 38

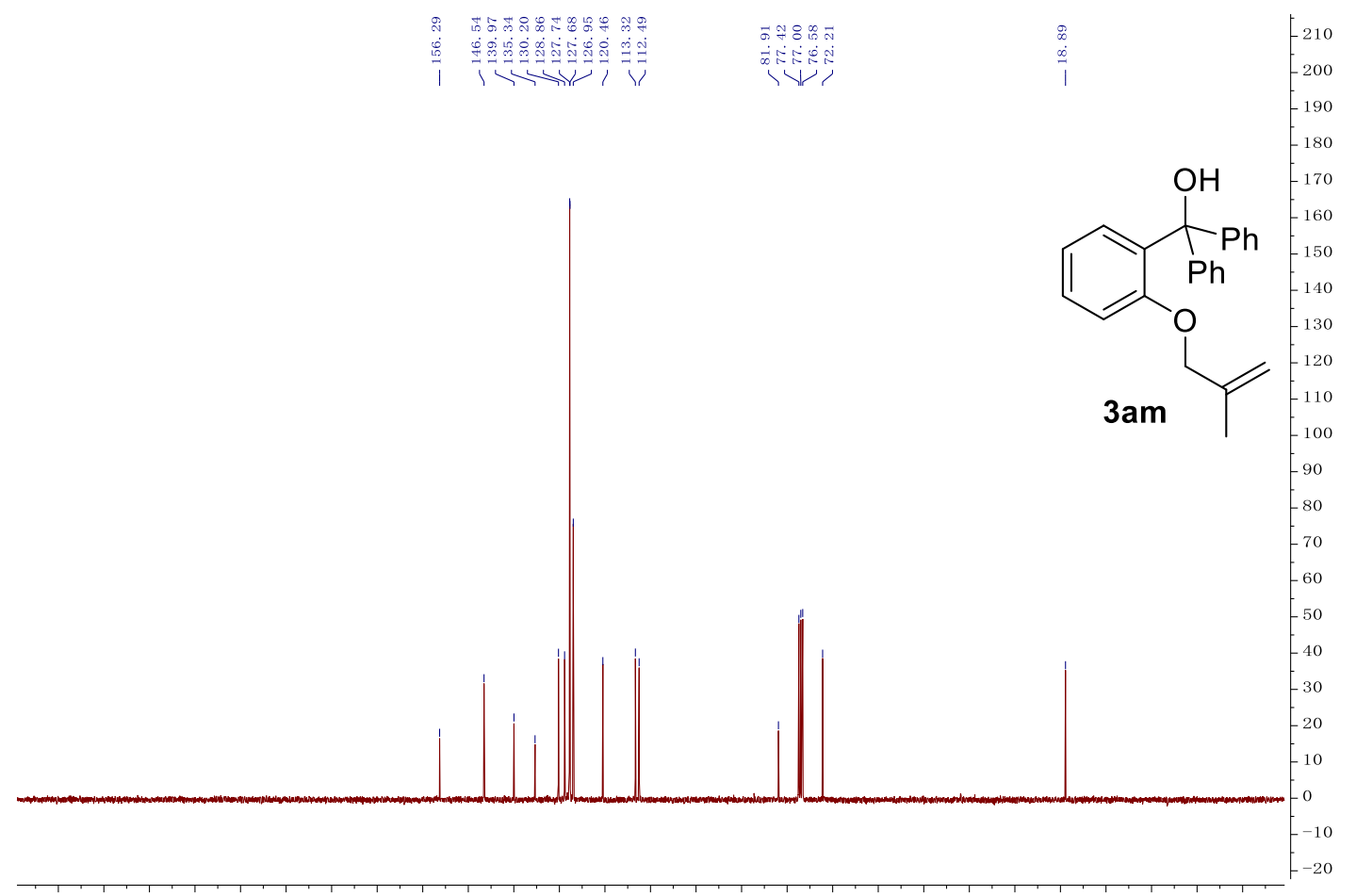

$\begin{array}{lllllllllllllllllllllllllllllllll}1 & 240 & 230 & 220 & 210 & 200 & 190 & 180 & 170 & 160 & 150 & 140 & 130 & 120 & 110 & 100 & 90 & 80 & 70 & 60 & 50 & 40 & 30 & 20 & 10 & 0 & -10 & -20\end{array}$ Mar17-2016. 571. fid - stu tang txj 254 - carbon CDC13/opt/topspin av1 38 